Article

\title{
Genetic Variation in Response to N, P, or K Deprivation in Baby Leaf Lettuce
}

\section{Ivan Simko}

Crop Improvement and Protection Research Unit, United States Department of Agriculture, Agricultural Research Service, Salinas, CA 93905, USA; ivan.simko@usda.gov

Received: 31 December 2019; Accepted: 13 February 2020; Published: 3 March 2020

check for updates

\begin{abstract}
Lettuce harvested at the baby leaf stage is a popular component of mixed salads in ready-to-use packages. Little is known, however, about response of baby leaf lettuce to nitrogen $(\mathrm{N})$, phosphorus $(\mathrm{P})$, and potassium $(\mathrm{K})$ fertilization. Eight lettuce accessions were subjected to five fertilization treatments to investigate genetic differences in reaction to $\mathrm{N}, \mathrm{P}$, and $\mathrm{K}$ fertilization. The control treatment provided optimal levels of macronutrients for plant growth, while other treatments deprived plants of either N, P, or K. Potassium deprivation had no obvious effect on plant weight or composition, apart from substantially decreased potassium content. Nitrogen and phosphorus deprivations, however, extensively decreased fresh weight and affected plant composition. Phosphorus and nitrogen deprivation considerably increased anthocyanin content in red-colored accessions, but anthocyanin was decreased in dark green-colored accessions, indicating differences in regulation of anthocyanin biosynthesis. Correlations between fresh weight, chlorophyll, anthocyanin, nitrogen, phosphorus, and potassium content were substantially affected by selection of datasets used for analyses; some relationships were revealed when analyzed separately by individual treatments, while others were more likely to be detected when analyzed by individual accessions. Absolute $\left(\Delta_{\mathrm{ABS}}\right)$ and relative $\left(2 \Delta_{\mathrm{REL}}\right)$ parameters described in this study were suitable for detecting overand underperforming accessions. The $\Delta_{\mathrm{ABS}}$ identified the absolute Lb-fold (logarithm to the base of 2, binary logarithm) change in performance of an accession in a treatment as compared to its performance in control conditions. The $2 \Delta_{\text {REL }}$ parameter showed relative Lb-fold change for an accession as compared to the overall mean of $\Delta_{\mathrm{ABS}}$ values of all accessions tested in control and treatment conditions.
\end{abstract}

Keywords: anthocyanin; chlorophyll; composition; Lactuca sativa; macronutrients; performance parameters

\section{Introduction}

Lettuce (Lactuca sativa L.) for baby leaf production is harvested approximately 30 days after planting when the first four true leaves reach a length of about 5-13 cm [1]. Baby leaf lettuce is the primary component of spring mix salads that are popular in ready-to-use packages [2]. Raw lettuce leaves contain dietary fiber, several important dietary minerals, vitamins (e.g., vitamins A, B9, and C), and bioactive compounds (e.g., chlorophyll, carotenoids, and phenolic compounds) [3-5] that contribute to human nutritional benefits [6]. Fertilization of lettuce with different rates of nitrogen $(\mathrm{N})$, phosphorus $(\mathrm{P})$, and potassium $(\mathrm{K})$ affects plant yield, nutritional quality $[7,8]$, postharvest quality [9,10], resistance to pathogens [11], microbiota in phyllosphere [12], and possibly also survival of human enteric pathogens on leaves [13]. In baby spinach, biomass yield, chlorophyll content, and leaf area increased significantly with increasing rates of $\mathrm{N}$ and $\mathrm{P}$; while $\mathrm{K}$ had no effect on the yield, chlorophyll content, stomatal conductance, and leaf area [14]. In lettuce, chlorophyll concentration increased with increasing levels of $\mathrm{N}[7,15]$. Anthocyanin accumulation in plants is most commonly 
associated with P deficiency [16], though deficiencies in $\mathrm{N}$ and $\mathrm{K}$ have also been noted to induce anthocyanin formation $[17,18]$.

Lettuce requires a sufficient supply of essential macronutrients for optimal production, which are frequently provided in the form of mineral fertilizers. To minimize the environmental impact and financial cost associated with using fertilizers, it is important to improve the efficiency with which macronutrients from fertilizers are used by plants [19]. Many of the modern crops have low fertilizer use efficiency, in particular nitrogen use efficiency, because they have been bred in the presence of non-limiting mineral fertilization conditions [20]. To develop breeding lines with high fertilizer use efficiency, it will be necessary to screen a range of genotypes for their responses to limited supply of N, P, and K, and to assess relationships between supplied macronutrients and plant composition. Genotypes that can produce high yield and maintain good quality at low levels of macronutrients may be selected for future studies and integrated into breeding programs focused on developing lettuces with improved fertilizer use efficiency. Although several studies were previously performed to investigate the effect of $\mathrm{N}, \mathrm{P}$, and $\mathrm{K}$ fertilization on yield and quality of leafy vegetables, data that describe genetic variation in response to mineral nutrition in baby leaf lettuce are lacking. Therefore, the objectives of this study were (1) to assess genetic differences among eight lettuce accessions in their response to N, P, or K deprivation at the baby leaf stage; and (2) to determine the relationship between plant fresh weight and content of chlorophyll, anthocyanin, N, P, and $\mathrm{K}$ in leaves at both optimal and suboptimal growing conditions. Four N, P, and K, deprivation treatments used in this study were aimed to markedly change plant composition, thus allowing a determination of how altered plant composition affected correlations between traits.

\section{Materials and Methods}

Five different treatments were used in the present study to investigate genetic differences in reaction to nutrients: one treatment provided optimal levels of macronutrients for plant growth; and four treatments where plants were deprived of either N, P, or K. Differences in the performance of accessions at four suboptimal conditions were analyzed using several methodologies, including an absolute $\left(\Delta_{\mathrm{ABS}}\right)$ parameter and a novel, relative $\left(2 \Delta_{\mathrm{REL}}\right)$ parameter that compares relative performances of all accessions under the particular treatment after adjusting for their performance under control conditions (optimal levels of macronutrients). In this study, the term "performance" is used to indicate the change in values of an accession with the particular fertilizer treatment as compared to the values measured on plants of the same accession grown under control conditions (i.e., changes in yield, and in anthocyanin, chlorophyll, N, P, and K contents).

\subsection{Plant Materials and Growth Conditions}

A diverse set of eight lettuce accessions evaluated in this study included seven cultivars, namely 'Annapolis' (romaine, dark red), 'Balady Barrage' (B. Barrage; stem type, green), 'Darkland' (romaine, dark green), 'Eruption' (Latin, red), 'La Brillante' (Batavia, light green), 'Merlot' (leaf type, dark red), 'Salinas' (iceberg, green), and a single accession of prickly lettuce UC96US23 (Lactuca serriola L., dark green), which is a species from which desirable traits and alleles can be introgressed into cultivated lettuce. Lettuce seeds were planted in potting soil (Premium Growers Mix, Sun Land Garden Products, Watsonville, CA, USA), covered with sand, and then watered. Trays with seeds were kept for $48 \mathrm{~h}$ at $10{ }^{\circ} \mathrm{C}$ in the dark to improve uniformity of germination. Afterwards, trays were transferred to a growth room with a temperature of $20^{\circ} \mathrm{C}$ and $16 \mathrm{~h} / 8 \mathrm{~h}$ light/dark photoperiod for germination and initial growth. Established plants of uniform development were transplanted to $7.6 \mathrm{~cm}$ pots $(\sim 514 \mathrm{~mL}$ volume) containing 1:2 mix of Espoma VM8 8-Quart Organic Vermiculite (Espoma, Millville, NJ, USA) and sand. Plants were fertilized with Non-Coated Ammonium Nitrate 34-0-0 Prill Form Fertilizer (Intermountain Farmers Association, Salt Lake City, UT, USA), Triple Super Phosphate 0-46-0 Easy Peasy Plants 99\% pure (Easy Peasy Plants, Alvin, IL, USA), and All-Natural Muriate of Potash-Easy Peasy 0-0-60 Potassium (Easy Peasy Plants, Alvin, IL, USA) to achieve concentrations of N, P, and K 
shown in Table 1. All plants were sprayed with an identical amount of micronutrient mix solution, which was prepared by dissolving $1 \mathrm{~g}$ of Axilo Mix 5 (0-0-0) Micronutrient Mix (Valagro USA, Houston, TX, USA) in $1 \mathrm{~L}$ of distilled water. Pots were then watered to saturation. Plants were grown in a greenhouse in a randomized complete block design with four replications $(8 \times 5 \times 4=160$ plants in total) and watered daily as needed. The average daily temperature in the greenhouse (April and May 2018) ranged from 20 to $24{ }^{\circ} \mathrm{C}$, daylength ranged from approximately 13 to $14 \mathrm{~h}$, and outdoor average daily light integrals were between 40 and $55 \mathrm{~mol} \mathrm{~m}^{-2} \mathrm{~d}^{-1}$. When four true leaves on the majority of control plants (NPK treatments) reached about $10 \mathrm{~cm}$, all plants were harvested. Each of the four individual plants collected per accession was used for quantification of compounds.

Table 1. Treatments and combinations of fertilizers.

\begin{tabular}{ccccc}
\hline Treatment & $\begin{array}{c}\text { Ammonium } \\
\text { Nitrate }^{\mathbf{z}} \mathbf{( g )}\end{array}$ & Phosphate $^{\mathbf{y}}(\mathbf{g})$ & Potash $^{\mathbf{x}}(\mathbf{g})$ & $\begin{array}{c}\text { Micronutrients } \\
\mathbf{w}(\mathbf{m L})\end{array}$ \\
\hline NPK (control) & 6 & 3 & 2.3 & 3 \\
nPK & 2 & 3 & 2.3 & 3 \\
PK & 0 & 3 & 2.3 & 3 \\
NK & 6 & 0 & 2.3 & 3 \\
NP & 6 & 3 & 0 & 3
\end{tabular}

\footnotetext{
${ }^{\mathrm{z}}$ Non-Coated Ammonium Nitrate 34-0-0 Prill Form; ${ }^{\mathrm{y}}$ Triple Super Phosphate 0-46-0 Easy Peasy Plants 99\% pure; ${ }^{x}$ All-Natural Muriate of Potash-Easy Peasy 0-0-60 Potassium; ${ }^{\mathrm{w}} 1 \mathrm{~g}$ of Axilo Mix 5 (0-0-0) Micronutrient Mix in 1L of distilled water.
}

\subsection{Quantification of Fresh Weigh, Pigments, and N, P, K}

Fresh weight of above ground parts was quantified immediately after harvest. The relative content of chlorophyll and anthocyanin was determined two days before harvest using SPAD-502 (Spectrum Technologies, Plainfield, IL, USA) and ACM-200 plus (Opti-Sciences, Hudson, NH, USA) hand-held meters, respectively. These devices use light transmittance to provide good in situ estimates of relative contents of the two pigments [21,22]. Chlorophyll and anthocyanin were measured on three leaves of similar age (avoiding youngest and oldest leaves) and size per plant. The measuring clip was positioned about $1 \mathrm{~cm}$ from the edge of the leaf, ensuring that major veins were avoided. The content of chlorophyll is expressed in SPAD units; the content of anthocyanin is expressed in anthocyanin content index (ACI) units. For each plant, the averages of three measurements of chlorophyll and anthocyanin were recorded and used in statistical analyses.

Leaf tissue of harvested plants was used for quantification of N, P, and K contents. All laboratory analyses were performed by UC Davis Analytical Laboratory (https://anlab.ucdavis.edu) using common quantification procedures developed for plant tissue samples. Prior to NPK analyses, samples were dried and ground to pass through a 40 -mesh screen $(<0.4 \mathrm{~mm})$ on the Wiley Mini-Mill (Thomas Scientific, Swedesboro, NJ, USA). N was quantified using a method that measures soluble nitrate $\left(\mathrm{NO}_{3}-\mathrm{N}\right)$ in plant materials based on an extraction with a solution of $2 \%$ acetic acid [23]. Nitrate was determined by a Quikchem 8500 Flow Injection Analysis (FIA) system (Lachat Instruments, Loveland, CO, USA) using the reduction to nitrite via a copperized cadmium column, diazotization with sulfanilamide, followed by coupling with $N$-(1-naphthyl) ethlyenediaminie dihydrochloride. The absorbance of the product was measured at $520 \mathrm{~nm}$. P was quantified using a method that determines the amount of phosphorus $\left(\mathrm{PO}_{4}-\mathrm{P}\right)$ in plant materials by extraction with a solution of $2 \%$ acetic acid [24]. Phosphorus concentration in the extract was determined spectrophotometrically by reacting with ammonium molybdate and antimony potassium tartrate under acidic conditions to form a complex. This complex was reduced with ascorbic acid to form a blue complex that absorbs light at $880 \mathrm{~nm}$. The absorbance is proportional to the concentration of phosphorus in the sample. Samples were analyzed using an automated Quikchem 8500 FIA system. K was quantified using a method that measures soluble potassium $(\mathrm{K})$ present in plant materials by extraction with a solution of $2 \%$ acetic acid [25]. Potassium was quantitatively determined in the extract using inductively coupled plasma 
atomic emission spectrometry (ICP-AES) with a Thermo Scientific iCAP 6000 (Waltham, MA, USA). N, $\mathrm{P}$, and $\mathrm{K}$ content are reported in $\mathrm{g}$ per $1 \mathrm{~kg}$ of fresh weight $\left(\mathrm{g} \mathrm{kg}^{-1} \mathrm{FW}\right)$.

\subsection{Data Analyses}

Three statistical approaches were used to compare the performance of eight accessions tested in five environments. The distinctions of those statistical approaches can be explained by a simplified example that considers only three accessions, namely $A, B$, and $C$, grown in control $\left(A_{C}=10, B_{C}=15, C_{C}=30\right)$ and treatment $\left(A_{T}=20, B_{T}=25, C_{T}=34\right)$ environments, where numerical values indicate the averages of their respective performances. Statistical analyses were performed on data from all accessions grown in all environments (e.g., $A_{C}, B_{C}, C_{C}, A_{T}, B_{T}, C_{T}$ ) using analysis of variance (ANOVA) followed by a multiple comparison procedure (Tukey's honest significant difference [HSD] test) to identify significant differences. However, because this kind of post hoc test compares all possible pairs of means, when eight accessions are tested in five environments, the approach leads to a very large number or pairwise comparisons $(8 \times 5 \times(8 \times 5-1) / 2=780)$ that affect the experiment-wise error rate. Moreover, testing of all paired comparisons is not always necessary or relevant (e.g., comparing performance of a cultivar grown in one environment with another cultivar grown in a different environment).

Two other statistical tests were performed on absolute $\left(\Delta_{\mathrm{ABS}}\right)$ and relative $\left(2 \Delta_{\mathrm{REL}}\right)$ differences in values detected for an accession grown using a specific treatment and control environment. Here, $\Delta_{\mathrm{ABS}}$ denotes an absolute difference in performance of an accession in two environments. Using the above example, the $\Delta_{A B S}$ for accession $A$ is calculated as: $A_{T}-A_{C}, 20-10=10$. Because only performance of a single accession in two environments is compared using this approach, the $\Delta_{\text {ABS }}$ parameter is independent from performances of other accessions tested in the same experiments. Statistical analyses of $\Delta_{\text {ABS }}$ significance in this study were performed using Student's $t$-test. Here, $2 \Delta_{\text {REL }}$ denotes a relative difference in performance of an accession and is calculated by comparing the $\Delta_{\mathrm{ABS}}$ of all accessions tested in the same two environments (control and a particular treatment). Therefore, this parameter shows a relative performance that is affected by the performance of other tested accessions. When the goal is to compare the $\Delta_{\mathrm{ABS}}$ of accession $\mathrm{A}$ to the overall mean of all $\Delta_{\mathrm{ABS}}$, the $2 \Delta_{\mathrm{REL}}$ for this accession is calculated as $\Delta_{\mathrm{ABS}}$ of $\mathrm{A}$ minus the average of $\Delta_{\mathrm{ABS}}$ of $\mathrm{A}, \Delta_{\mathrm{ABS}}$ of $\mathrm{B}$, and $\Delta_{\mathrm{ABS}}$ of $\mathrm{C}$. Using the above example, $2 \Delta_{\text {REL }}$ for A equals $(20-10)-((20-10)+(25-15)+(34-30)) / 3=2$. The sum of all $2 \Delta_{\text {REL }}$ values equals zero. The $2 \Delta_{\mathrm{REL}}$ values detected in this study were statistically evaluated using analysis of means (ANOM). Alternative statistical analyses of $\Delta_{\mathrm{ABS}}$ values could be performed using multiple comparisons procedures.

Pairwise correlations between values of six tested traits were performed on 14 datasets to identify how treatments and genotypes affect relationships among traits. Eight of the datasets comprised data from a single accession each, tested for all five treatments. These datasets show how correlations differ among accessions tested across all environments. Five of the datasets comprised data from a single treatment each, but using all eight accessions. These datasets show how correlations differ among treatments when data from all accessions are considered. Analyses were also performed on the complete dataset, which included all combinations of tested treatments and accessions, to identify correlations that could be detected in combined data.

Principal component analysis (PCA) of traits was done using the correlation matrix as an input dataset. Effect size $\left(\omega^{2}\right)$, which estimates how much variance in the response variable is accounted for by individual factors (accessions and treatments) and their interaction, was calculated from ANOVA output [26]. All statistical analyses were performed using JMP software v. 14.2.0 (SAS Institute, Cary, NC, USA) and Microsoft Excel for Mac v. 16.16.5 (Microsoft, Redmond, WA, USA).

\subsection{Data Transformation}

It has been previously observed that lognormal distribution in plant weight develops naturally [27], and therefore assumption of lognormality of this trait is probably appropriate in most applications [28]. Logarithmic transformation of weight and other traits is not performed only because of statistical 
convenience, but it is appropriate due to the fundamentally multiplicative nature of biological phenomena [29]. In the present study, data for all six traits were statistically analyzed using original (measured) values and also logarithmically transformed values (logarithm to the base of 2, binary logarithm, Lb). Because statistical power for all but one trait was greater when using Lb-transformed values, only these results are presented in this paper (original values are shown in Supplementary Table S1). The only exception was the SPAD index, which showed practically identical statistical power when using both the original and the transformed values. However, to keep uniformity of data across all traits, only results obtained using Lb-transformed SPAD values are shown. "Lb" was added in front of trait names to indicate that data were logarithmically transformed ( $\mathrm{Lb}$ FW, Lb SPAD, Lb ACI, Lb N, $\mathrm{Lb} \mathrm{P}$, and $\mathrm{Lb} \mathrm{K}$ ). Use of logarithmic transformation means that the difference between two "Lb" values is the logarithm of the ratio of two original values (e.g., $\left.\mathrm{Lb}\left(\mathrm{A}_{\mathrm{T}}\right)-\mathrm{Lb}\left(\mathrm{A}_{\mathrm{C}}\right)=\mathrm{Lb}\left(\mathrm{A}_{\mathrm{T}} / \mathrm{A}_{\mathrm{C}}\right)\right)$. Therefore,

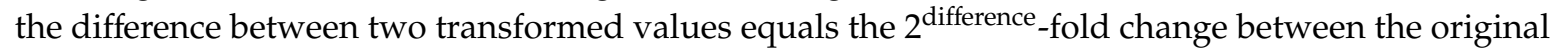
values (e.g., for $A_{T}=20$ and $A_{C}=10, L b 20-\mathrm{Lb} 10=1$, which in turn is $2^{1}=2$-fold change from $A_{C}=10$ to $A_{T}=20$, or $20 / 10=2$ ). The use of transformed values also means that back transformation of averages calculated from logarithms equals geometric means calculated from original values.

\section{Results}

\subsection{Effect of Treatments}

Plants of eight accessions were grown at control (NPK) conditions, with all nutrients provided at an optimal level and four treatments having a limited amount of nitrogen (nPK and PK), phosphorus (NK), and potassium (NP), with treatments having a profound effect on six analyzed traits. Results of two-way ANOVA (Table 2) indicate that compared to control, partial N restriction (nPK) led to lower fresh weight (Lb FW) and lower content of $\mathrm{N}(\mathrm{Lb} \mathrm{N})$. Total $\mathrm{N}$ deprivation $(\mathrm{PK})$ decreased values of all six tested traits: plant fresh weight ( $\mathrm{Lb}$ FW), content of chlorophyll (Lb SPAD), anthocyanin ( $\mathrm{Lb} \mathrm{ACI})$, nitrogen ( $\mathrm{Lb} \mathrm{N}$ ), phosphorus ( $\mathrm{Lb} \mathrm{P})$, and potassium (Lb K). P deprivation (NK) reduced the fresh weight (Lb FW), content of chlorophyll (Lb SPAD), phosphorus (Lb P), and potassium (Lb $\mathrm{K})$, but increased the content of anthocyanin (Lb ACI) and nitrogen ( $\mathrm{Lb} \mathrm{N}$ ). K deprivation (NP) did not show any significant effect on change of tested traits compared to control, with the exception of potassium content $(\mathrm{Lb} \mathrm{K})$, which was decreased. PCA revealed that data from NK and PK treatments were generally well separated from data of the other three treatments and from each other (Figure 1a). Overall, there was a close relationship detected between Lb SPAD and Lb N traits (Figure 1b).

\subsection{Fresh Weight}

Fresh weight was influenced by both accessions and nutrient treatment. Fresh weight, expressed as a binary logarithm of FW in $\mathrm{g}$ (Lb FW), ranged from 0.80 for UC96US23 under PK conditions to 5.93 for 'Darkland' under NPK conditions (Table 3). The overall Lb FW mean for accessions was lowest for UC96US23 (2.63) and highest for 'Darkland' (4.16), while the overall mean for treatments ranged from 1.60 under PK conditions to 5.03 under NPK conditions (Table 2). A large effect size was found for treatments $\left(\omega^{2}=0.852\right)$, while small effect size was found for accessions $\left(\omega^{2}=0.076\right)$ and treatment by accession interaction $\left(\omega^{2}=0.009\right)$. All accessions had significant negative $\Delta_{\mathrm{ABS}}$ under PK and NK treatments. B. Barrage and 'Darkland' also had significant negative $\Delta_{\text {ABS }}$ values with nPK. No significant $\Delta_{\mathrm{ABS}}$ for Lb FW was detected with NP (Figure 2). The $2 \Delta_{\mathrm{REL}}$ analysis revealed that fresh weight of 'Darkland' declined more than in other tested accessions with PK treatment. 
Table 2. Main effect of factors, calculated from binary logarithm (Lb) values, on fresh weight (Lb FW) and contents of chlorophyll (Lb SPAD), anthocyanin (Lb ACI), nitrogen ( $\mathrm{Lb} \mathrm{N}$ ), phosphorus (Lb P), and potassium ( $\mathrm{Lb} \mathrm{K})$.

\begin{tabular}{|c|c|c|c|c|c|c|}
\hline Descriptor & Lb FW & Lb SPAD & Lb ACI & $\mathbf{L b} \mathbf{N}$ & Lb P & Lb K \\
\hline Effect Size & \multicolumn{6}{|c|}{$\omega^{2 z}$} \\
\hline Model & $0.937^{* * *}$ & $0.726 * * *$ & $0.908^{* * *}$ & $0.894^{* * *}$ & $0.922 * * *$ & $0.847^{* * *}$ \\
\hline Accession & $0.076^{* * *}$ & $0.377^{* * *}$ & $0.725^{* * *}$ & $0.108^{* * *}$ & $0.031^{* * *}$ & $0.320^{* * *}$ \\
\hline Treatment & $0.852 * * *$ & $0.316^{* * *}$ & $0.037^{* * *}$ & $0.759^{* * *}$ & $0.892 * * *$ & $0.435^{* * *}$ \\
\hline Interaction & $0.009 *$ & $0.032 *$ & $0.146^{* * *}$ & $0.027^{* * *}$ & 0.000 & $0.092^{* * *}$ \\
\hline Accessions & \multicolumn{6}{|c|}{ Mean ${ }^{y}$} \\
\hline Annapolis & $3.68 \mathrm{~b}$ & $5.08 \mathrm{ab}$ & $5.48 \mathrm{a}$ & $1.88 \mathrm{bc}$ & $-1.21 \mathrm{ab}$ & $1.79 \mathrm{~b}$ \\
\hline B. Barrage & $3.88 \mathrm{ab}$ & $4.83 \mathrm{~b}$ & $2.30 \mathrm{f}$ & $1.54 \mathrm{~d}$ & $-1.65 c$ & $1.47 \mathrm{c}$ \\
\hline Darkland & $4.16 \mathrm{a}$ & $5.01 \mathrm{~b}$ & $2.73 \mathrm{de}$ & $1.50 \mathrm{~d}$ & $-1.49 b c$ & $1.57 \mathrm{c}$ \\
\hline Eruption & $4.00 \mathrm{ab}$ & $4.92 \mathrm{~b}$ & $4.26 \mathrm{~b}$ & $1.84 \mathrm{c}$ & $-1.16 \mathrm{a}$ & $2.24 \mathrm{a}$ \\
\hline La Brillante & $3.86 \mathrm{ab}$ & $4.40 \mathrm{c}$ & $1.75 \mathrm{~g}$ & $2.07 \mathrm{ab}$ & $-1.09 \mathrm{a}$ & $2.14 \mathrm{a}$ \\
\hline Merlot & $3.82 \mathrm{ab}$ & $4.32 \mathrm{c}$ & $3.73 \mathrm{c}$ & $1.95 \mathrm{bc}$ & $-1.03 \mathrm{a}$ & $2.11 \mathrm{a}$ \\
\hline Salinas & $3.75 \mathrm{~b}$ & $4.93 \mathrm{~b}$ & 2.39 ef & $1.94 \mathrm{bc}$ & $-1.26 a b$ & $2.12 \mathrm{a}$ \\
\hline UC96US23 & $2.63 \mathrm{c}$ & $5.28 \mathrm{a}$ & $2.96 \mathrm{~d}$ & $2.18 \mathrm{a}$ & $-1.17 \mathrm{a}$ & $2.16 \mathrm{a}$ \\
\hline Treatments & \multicolumn{6}{|c|}{ Mean } \\
\hline NPK & $5.03 \mathrm{a}$ & $5.02 \mathrm{a}$ & $3.06 \mathrm{bc}$ & $2.16 \mathrm{~b}$ & $-0.60 \mathrm{a}$ & $2.22 \mathrm{a}$ \\
\hline $\mathrm{nPK}$ & $4.53 \mathrm{~b}$ & $4.94 \mathrm{ab}$ & $3.07 \mathrm{bc}$ & $1.80 \mathrm{c}$ & $-0.71 \mathrm{a}$ & $2.28 \mathrm{a}$ \\
\hline PK & $1.60 \mathrm{~d}$ & $4.32 \mathrm{c}$ & $2.94 \mathrm{c}$ & $0.78 \mathrm{~d}$ & $-1.26 b$ & $1.95 \mathrm{~b}$ \\
\hline NK & $2.42 \mathrm{c}$ & $4.85 \mathrm{~b}$ & $3.70 \mathrm{a}$ & $2.36 \mathrm{a}$ & $-3.18 c$ & $1.94 \mathrm{~b}$ \\
\hline NP & $5.02 \mathrm{a}$ & $5.11 \mathrm{a}$ & $3.23 \mathrm{~b}$ & $2.21 \mathrm{~b}$ & $-0.53 \mathrm{a}$ & $1.36 \mathrm{c}$ \\
\hline
\end{tabular}

z Effect size $\left(\omega^{2}\right)$ and significance of factors were calculated from two-way ANOVA. Asterisks indicate value significance at $p \leq 0.05\left(^{*}\right), p \leq 0.01\left(^{* *}\right)$, and $p \leq 0.001\left(^{* * *}\right)$. ${ }^{\mathrm{y}}$ Values for accessions are means of 20 plants, while values for treatments are means of 32 plants. Mean values within a column and a factor followed by different letters are significantly different at $p \leq 0.05$ using the Tukey's HSD test.

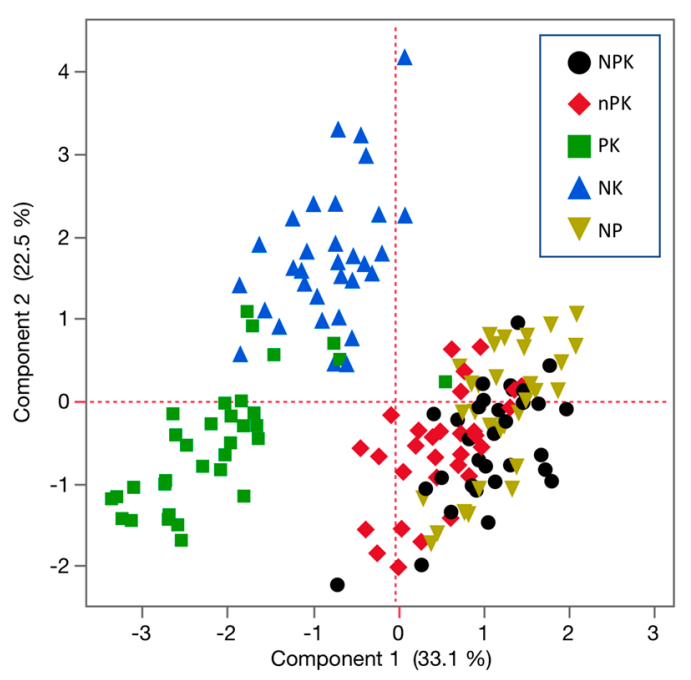

(a)

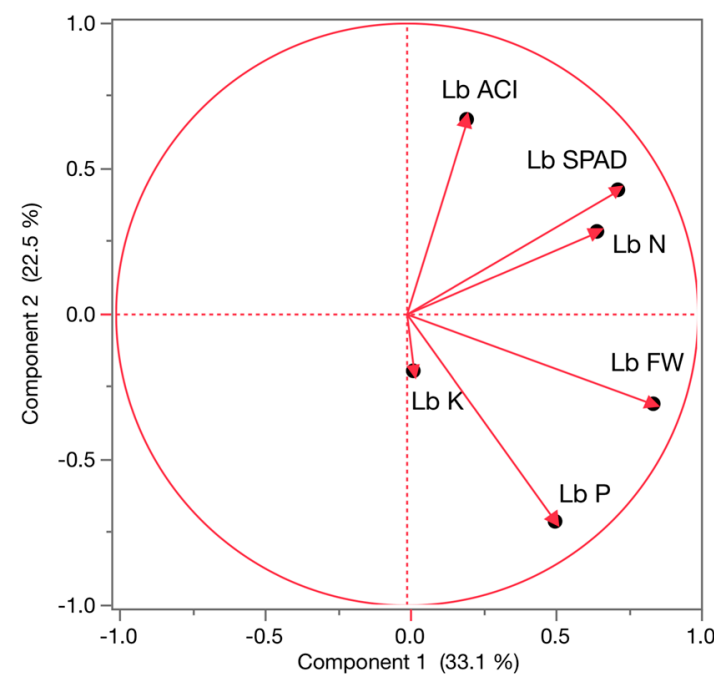

(b)

Figure 1. Principal component analysis score plot (a) and loading plot (b). Data from five treatments are indicated by icons of different shapes and colors. NPK treatment (control) supplied optimal concentrations of all three macronutrients, nPK treatment supplied only $1 / 3$ of $\mathrm{N}$ compared to control, PK treatment did not supply N, NK treatment did not supply P, and NP treatment did not supply K. NK and PK treatment data are generally well separated from those of the other three treatments and from each other. The loading plot results indicate a close relationship between Lb SPAD (chlorophyll content) and $\mathrm{Lb} \mathrm{N}$ (nitrogen content) traits. 
Table 3. Combined effect of factors on fresh weight (Lb FW), content of chlorophyll (Lb SPAD), anthocyanin ( $\mathrm{Lb} \mathrm{ACI})$, nitrogen ( $\mathrm{Lb} \mathrm{N})$, phosphorus ( $\mathrm{Lb} \mathrm{P})$, and potassium ( $\mathrm{Lb} \mathrm{K})$.

\begin{tabular}{|c|c|c|c|c|c|c|c|}
\hline Accession & Treatment $^{\mathrm{z}}$ & Lb FW & Lb SPAD & Lb ACI & Lb N & Lb P & Lb K \\
\hline Annapolis & NPK & $4.97 \mathrm{a}-\mathrm{e}^{\mathrm{y}}$ & $5.28 \mathrm{a}-\mathrm{f}$ & $4.69 \mathrm{~b}-\mathrm{e}$ & $2.17 b-g$ & $-0.62 \mathrm{a}-\mathrm{d}$ & $2.12 \mathrm{a}-\mathrm{i}$ \\
\hline B. Barrage & NPK & $5.37 \mathrm{a}-\mathrm{d}$ & $5.15 \mathrm{a}-\mathrm{g}$ & $2.62 \mathrm{j}-\mathrm{o}$ & $1.59 \mathrm{~g}-\mathrm{j}$ & $-1.11 b-f$ & $1.76 \mathrm{~g}-\mathrm{m}$ \\
\hline Darkland & NPK & $5.93 \mathrm{a}$ & $5.31 \mathrm{a}-\mathrm{e}$ & $3.07 \mathrm{~h}-1$ & $1.77 \mathrm{e}-\mathrm{i}$ & $-0.74 \mathrm{a}-\mathrm{f}$ & $1.87 \mathrm{e}-\mathrm{m}$ \\
\hline Eruption & NPK & $5.13 \mathrm{a}-\mathrm{d}$ & $5.06 \mathrm{a}-\mathrm{g}$ & $3.63 \mathrm{e}-\mathrm{j}$ & $2.21 \mathrm{a}-\mathrm{f}$ & $-0.52 a-c$ & $2.21 \mathrm{a}-\mathrm{h}$ \\
\hline La Brillante & NPK & $5.02 \mathrm{a}-\mathrm{d}$ & $4.72 \mathrm{c}-\mathrm{j}$ & $1.93 \mathrm{~m}-\mathrm{p}$ & $2.54 \mathrm{a}-\mathrm{c}$ & $-0.33 a b$ & $2.57 \mathrm{a}$ \\
\hline Merlot & NPK & $4.86 \mathrm{a}-\mathrm{f}$ & $4.28 \mathrm{~h}-1$ & $2.80 \mathrm{i}-\mathrm{n}$ & $2.37 \mathrm{a}-\mathrm{e}$ & $-0.21 \mathrm{a}$ & $2.43 \mathrm{a}-\mathrm{d}$ \\
\hline Salinas & NPK & $5.09 \mathrm{a}-\mathrm{d}$ & $5.01 \mathrm{a}-\mathrm{h}$ & $2.45 \mathrm{k}-\mathrm{p}$ & $2.22 \mathrm{a}-\mathrm{f}$ & $-0.63 \mathrm{a}-\mathrm{d}$ & $2.41 \mathrm{a}-\mathrm{e}$ \\
\hline UC96US23 & NPK & $3.90 \mathrm{e}-\mathrm{h}$ & $5.39 \mathrm{a}-\mathrm{d}$ & $3.29 \mathrm{~g}-\mathrm{k}$ & $2.38 \mathrm{a}-\mathrm{d}$ & $-0.61 \mathrm{a}-\mathrm{d}$ & $2.36 \mathrm{a}-\mathrm{f}$ \\
\hline Annapolis & nPK & $4.43 c-g$ & $5.13 \mathrm{a}-\mathrm{g}$ & $4.96 \mathrm{~b}-\mathrm{d}$ & $1.86 \mathrm{~d}-\mathrm{i}$ & $-0.60 \mathrm{a}-\mathrm{d}$ & $1.97 \mathrm{c}-1$ \\
\hline B. Barrage & $\mathrm{nPK}$ & $4.27 \mathrm{~d}-\mathrm{g}$ & $5.01 \mathrm{a}-\mathrm{h}$ & $2.44 \mathrm{k}-\mathrm{p}$ & $1.55 \mathrm{~h}-\mathrm{j}$ & $-1.02 \mathrm{a}-\mathrm{f}$ & $1.84 \mathrm{f}-\mathrm{m}$ \\
\hline Darkland & $\mathrm{nPK}$ & $4.69 \mathrm{~b}-\mathrm{g}$ & $5.16 \mathrm{a}-\mathrm{g}$ & $2.61 \mathrm{j}-\mathrm{O}$ & $1.36 \mathrm{i}-\mathrm{k}$ & $-0.90 a-f$ & $2.20 \mathrm{a}-\mathrm{h}$ \\
\hline Eruption & $\mathrm{nPK}$ & $4.92 \mathrm{a}-\mathrm{f}$ & $5.05 \mathrm{a}-\mathrm{g}$ & $4.00 \mathrm{~d}-\mathrm{h}$ & $1.72 \mathrm{f}-\mathrm{i}$ & $-0.69 \mathrm{a}-\mathrm{e}$ & $2.55 \mathrm{ab}$ \\
\hline La Brillante & nPK & $4.68 \mathrm{~b}-\mathrm{g}$ & $4.46 \mathrm{~g}-1$ & $1.69 n-p$ & $1.96 c-h$ & $-0.59 \mathrm{a}-\mathrm{d}$ & $2.50 \mathrm{a}-\mathrm{c}$ \\
\hline Merlot & $\mathrm{nPK}$ & $4.66 \mathrm{~b}-\mathrm{g}$ & $4.22 \mathrm{i}-1$ & $3.13 \mathrm{~h}-1$ & $1.98 \mathrm{c}-\mathrm{h}$ & $-0.53 a-c$ & $2.42 \mathrm{a}-\mathrm{d}$ \\
\hline Salinas & nPK & $4.72 \mathrm{~b}-\mathrm{g}$ & $5.09 \mathrm{a}-\mathrm{g}$ & $2.61 \mathrm{j}-\mathrm{o}$ & $1.82 \mathrm{~d}-\mathrm{i}$ & $-0.79 \mathrm{a}-\mathrm{f}$ & $2.42 \mathrm{a}-\mathrm{d}$ \\
\hline UC96US23 & $\mathrm{nPK}$ & $3.83 \mathrm{f}-\mathrm{i}$ & $5.44 \mathrm{a}-\mathrm{c}$ & $3.16 \mathrm{~h}-1$ & $2.15 \mathrm{~b}-\mathrm{h}$ & $-0.58 \mathrm{a}-\mathrm{d}$ & $2.33 \mathrm{a}-\mathrm{f}$ \\
\hline Annapolis & PK & $1.43 \mathrm{~m}-\mathrm{n}$ & $4.55 \mathrm{f}-\mathrm{k}$ & $5.78 \mathrm{~b}$ & $1.11 \mathrm{j}-1$ & $-1.06 \mathrm{a}-\mathrm{f}$ & $2.14 \mathrm{a}-\mathrm{i}$ \\
\hline B. Barrage & PK & $2.10 \mathrm{k}-\mathrm{m}$ & $4.15 \mathrm{j}-1$ & $1.50 \mathrm{o}-\mathrm{p}$ & $0.39 \mathrm{~m}-\mathrm{n}$ & $-1.57 \mathrm{f}$ & $1.41 \mathrm{~m}-\mathrm{o}$ \\
\hline Darkland & PK & $1.85 \mathrm{k}-\mathrm{n}$ & $4.13 \mathrm{j}-1$ & $1.72 \mathrm{n}-\mathrm{p}$ & $0.28 \mathrm{n}$ & -1.49 ef & $1.17 \mathrm{n}-\mathrm{p}$ \\
\hline Eruption & PK & $1.781-\mathrm{n}$ & $4.46 \mathrm{~g}-1$ & $4.59 \mathrm{c}-\mathrm{f}$ & $0.88 \mathrm{k}-\mathrm{m}$ & $-1.06 \mathrm{a}-\mathrm{f}$ & $2.60 \mathrm{a}$ \\
\hline La Brillante & PK & $1.40 \mathrm{~m}-\mathrm{n}$ & $3.95 \mathrm{k}-1$ & $1.38 \mathrm{p}$ & $0.88 \mathrm{k}-\mathrm{n}$ & $-1.21 \mathrm{c}-\mathrm{f}$ & $1.92 \mathrm{~d}-\mathrm{m}$ \\
\hline Merlot & PK & $1.711-\mathrm{n}$ & 3.821 & $4.42 \mathrm{c}-\mathrm{g}$ & $0.91 \mathrm{k}-\mathrm{m}$ & $-1.08 \mathrm{~b}-\mathrm{f}$ & $2.30 \mathrm{a}-\mathrm{g}$ \\
\hline Salinas & PK & $1.741-\mathrm{n}$ & $4.66 \mathrm{~d}-\mathrm{k}$ & $2.041-\mathrm{p}$ & $0.751-\mathrm{n}$ & $-1.39 \mathrm{~d}-\mathrm{f}$ & $1.90 \mathrm{~d}-\mathrm{m}$ \\
\hline UC96US23 & PK & $0.80 n$ & $4.81 \mathrm{~b}-\mathrm{j}$ & $2.111-\mathrm{p}$ & $1.07 \mathrm{j}-1$ & $-1.26 c-f$ & $2.19 a-h$ \\
\hline Annapolis & NK & $2.31 \mathrm{k}-\mathrm{m}$ & $5.09 \mathrm{a}-\mathrm{g}$ & $7.08 \mathrm{a}$ & $2.21 \mathrm{a}-\mathrm{f}$ & $-3.22 \mathrm{~g}$ & $1.74 \mathrm{~h}-\mathrm{m}$ \\
\hline B. Barrage & NK & $2.54 \mathrm{k}-1$ & $4.60 \mathrm{e}-\mathrm{k}$ & $2.041-\mathrm{p}$ & $2.22 \mathrm{a}-\mathrm{f}$ & $-3.65 \mathrm{~g}$ & $1.48 \mathrm{l}-\mathrm{O}$ \\
\hline Darkland & NK & $2.77 \mathrm{i}-1$ & $4.98 \mathrm{a}-\mathrm{h}$ & $2.76 j-n$ & $2.26 \mathrm{a}-\mathrm{f}$ & $-3.56 \mathrm{~g}$ & $1.51 \mathrm{k}-\mathrm{n}$ \\
\hline Eruption & NK & $2.88 \mathrm{~h}-\mathrm{k}$ & $4.95 \mathrm{a}-\mathrm{h}$ & $5.26 \mathrm{bc}$ & $2.21 \mathrm{a}-\mathrm{f}$ & $-3.03 \mathrm{~g}$ & $2.33 \mathrm{a}-\mathrm{f}$ \\
\hline La Brillante & NK & $2.73 \mathrm{j}-1$ & $4.45 \mathrm{~g}-1$ & $1.92 \mathrm{~m}-\mathrm{p}$ & $2.73 \mathrm{ab}$ & $-2.89 \mathrm{~g}$ & $2.06 a-j$ \\
\hline Merlot & NK & $2.91 \mathrm{~h}-\mathrm{k}$ & $4.58 \mathrm{e}-\mathrm{k}$ & $5.22 \mathrm{bc}$ & $2.12 \mathrm{c}-\mathrm{h}$ & $-3.00 \mathrm{~g}$ & $1.94 \mathrm{~d}-\mathrm{m}$ \\
\hline Salinas & NK & $2.37 \mathrm{k}-\mathrm{m}$ & $4.98 \mathrm{a}-\mathrm{h}$ & $2.48 \mathrm{j}-\mathrm{p}$ & $2.36 \mathrm{a}-\mathrm{e}$ & $-3.26 \mathrm{~g}$ & $2.03 \mathrm{~b}-\mathrm{k}$ \\
\hline UC96US23 & NK & $0.89 n$ & $5.16 \mathrm{a}-\mathrm{g}$ & $2.81 \mathrm{i}-\mathrm{n}$ & $2.78 \mathrm{a}$ & $-2.85 \mathrm{~g}$ & $2.40 \mathrm{a}-\mathrm{e}$ \\
\hline Annapolis & $\mathrm{NP}$ & $5.27^{\mathrm{a}-\mathrm{d}}$ & $5.37 \mathrm{a}-\mathrm{d}$ & $4.88 \mathrm{~b}-\mathrm{d}$ & $2.04 \mathrm{c}-\mathrm{h}$ & $-0.55 \mathrm{a}-\mathrm{d}$ & $0.96^{\text {op }}$ \\
\hline B. Barrage & $\mathrm{NP}$ & $5.12^{\mathrm{a}-\mathrm{d}}$ & $5.27 \mathrm{a}-\mathrm{f}$ & $2.89 \mathrm{~h}-\mathrm{m}$ & $1.96 \mathrm{c}-\mathrm{i}$ & $-0.91 \mathrm{a}-\mathrm{f}$ & $0.84^{\mathrm{P}}$ \\
\hline Darkland & NP & $5.53^{\mathrm{ab}}$ & $5.49 \mathrm{ab}$ & $3.49 \mathrm{f}-\mathrm{k}$ & $1.86 \mathrm{~d}-\mathrm{i}$ & $-0.74 \mathrm{a}-\mathrm{f}$ & $1.10^{n-p}$ \\
\hline Eruption & $\mathrm{NP}$ & $5.31^{\mathrm{a}-\mathrm{d}}$ & $5.10 \mathrm{a}-\mathrm{g}$ & $3.84 \mathrm{~d}-\mathrm{i}$ & $2.19 a-g$ & $-0.50 a-c$ & $1.50 \mathrm{k}-\mathrm{O}$ \\
\hline La Brillante & NP & $5.44^{a-c}$ & $4.44^{\mathrm{g}-1}$ & $1.83 \mathrm{~m}-\mathrm{p}$ & $2.23 \mathrm{a}-\mathrm{f}$ & $-0.44 a-c$ & $1.63 \mathrm{i}-\mathrm{n}$ \\
\hline Merlot & NP & $4.94^{\mathrm{a}-\mathrm{e}}$ & $4.70^{\mathrm{d}-\mathrm{j}}$ & $3.10 \mathrm{~h}-1$ & $2.38 \mathrm{a}-\mathrm{d}$ & $-0.31 \mathrm{ab}$ & $1.47 \mathrm{l}-\mathrm{O}$ \\
\hline Salinas & NP & $4.82^{b-f}$ & $4.91^{\mathrm{a}-\mathrm{i}}$ & $2.38 \mathrm{k}-\mathrm{p}$ & $2.55 \mathrm{a}-\mathrm{c}$ & $-0.23 \mathrm{a}$ & $1.86 \mathrm{f}-\mathrm{m}$ \\
\hline UC96US23 & $\mathrm{NP}$ & $3.73^{g-j}$ & $5.58^{a}$ & $3.45 \mathrm{f}-\mathrm{k}$ & $2.50 \mathrm{a}-\mathrm{c}$ & $-0.56 \mathrm{a}-\mathrm{d}$ & $1.54 \mathrm{j}-\mathrm{n}$ \\
\hline
\end{tabular}

${ }^{\mathrm{z}}$ NPK treatment (control) supplied optimal concentrations of all three macronutrients; nPK treatment supplied only $1 / 3$ of N compared to control; PK treatment did not supply N; NK treatment did not supply P; and NP treatment did not supply K. ${ }^{y}$ Values are means of four plants (replicates). Mean values within a column followed by different letters are significantly different at $p \leq 0.05$ using the Tukey's HSD test.

\subsection{Chlorophyll Content Index (SPAD)}

The chlorophyll content index, expressed as the binary logarithm of SPAD (Lb SPAD), ranged from 3.82 for 'Merlot' with PK to 5.58 for UC96US23 with NP (Table 3). The overall Lb SPAD mean for accessions was lowest for 'Merlot' (4.32) and highest for UC96US23 (5.28), while the overall mean for treatments ranged from 4.32 with PK to 5.11 with NP (Table 2). Effect size was similar for accessions $\left(\omega^{2}=0.377\right)$ and treatments $\left(\omega^{2}=0.316\right)$, but substantially smaller for interaction $\left(\omega^{2}=0.032\right)$. Significant negative $\Delta_{\mathrm{ABS}}$ was detected for all accessions with PK, with the exception of 'Salinas', and also for B. Barrage with NK. Significant positive $\Delta_{\mathrm{ABS}}$, indicating an increase in 
chlorophyll index, was detected for 'Merlot' with NP. No $2 \Delta_{\text {REL }}$ showed significant difference from zero (Figure 3).

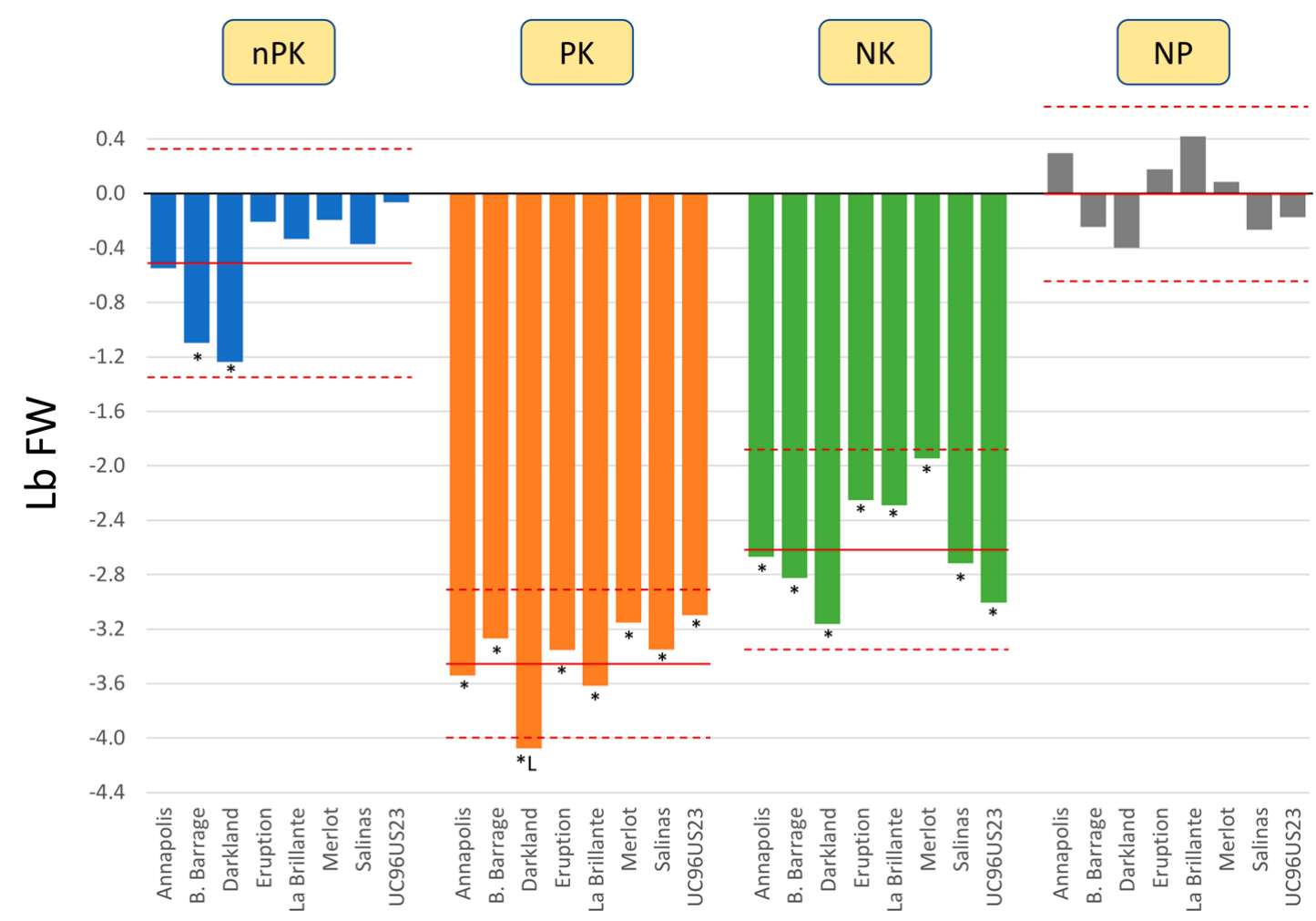

Figure 2. Values of $\Delta_{\mathrm{ABS}}$ and $2 \Delta_{\mathrm{REL}}$ parameters for fresh weight (Lb FW). The $\Delta_{\mathrm{ABS}}$ bars illustrate (Lb-fold) changes from values observed under control conditions (NPK treatment) for each accession and treatment. NPK treatment (control) supplied optimal concentrations of all three macronutrients, nPK treatment supplied only $1 / 3$ of $\mathrm{N}$ compared to control, PK treatment did not supply N, NK treatment did not supply $P$, and NP treatment did not supply K. Significant $(P \leq 0.05) \Delta_{\text {ABS }}$ values detected by Student's $t$-test are indicated by asterisks. Solid horizontal red lines show overall mean of $\Delta_{\mathrm{ABS}}$ at each treatment. Dashed horizontal red lines show $95 \%$ upper and lower decision limits for analysis of means (ANOM). The $2 \Delta_{\text {REL }}$ parameter is calculated as the difference between $\Delta_{\mathrm{ABS}}$ of an accession and the overall mean of $\Delta_{\mathrm{ABS}}$ for all accessions tested with the particular treatment. The significance of the $2 \Delta_{\text {REL }}$ parameter is indicated by capital letters $\mathrm{L}$ (significantly lower than overall mean) or $\mathrm{H}$ (significantly higher than overall mean).

\subsection{Anthocyanin Content Index (ACI)}

The anthocyanin content index, expressed as the binary logarithm of $\mathrm{ACI}$ ( $\mathrm{Lb} \mathrm{ACI})$, ranged from 1.38 for 'La Brillante' with PK to 7.08 for 'Annapolis' with NK (Table 3). The overall Lb ACI mean for accessions was lowest for 'La Brillante' (1.75) and highest for 'Annapolis' (5.48), while the overall mean for treatments ranged from 2.94 with PK to 3.70 with NK (Table 2). The effect size was high for accessions $\left(\omega^{2}=0.725\right)$ but low for treatments $\left(\omega^{2}=0.037\right)$. Lb ACI had the largest effect size for interaction $\left(\omega^{2}=0.146\right)$ from all tested traits. Significant positive $\Delta_{\text {ABS }}$ was detected for 'Annapolis', 'Eruption', and 'Merlot' with both PK and NK, indicating that anthocyanin content in these accessions increased as compared to the control (NPK). In comparison, significant negative $\Delta_{\mathrm{ABS}}$ was detected for B. Barrage, 'Darkland', and UC96US23 with PK, and for B. Barrage with NK (Figure 4). All accession-treatment combinations with significant $\Delta_{\mathrm{ABS}}$ values also had significant $2 \Delta_{\text {REL }}$ values. In addition, $2 \Delta_{\text {REL }}$ values for 'Darkland' and UC96US23 with NK were also significantly lower than the overall mean of $\Delta_{\mathrm{ABS}}$ values. 


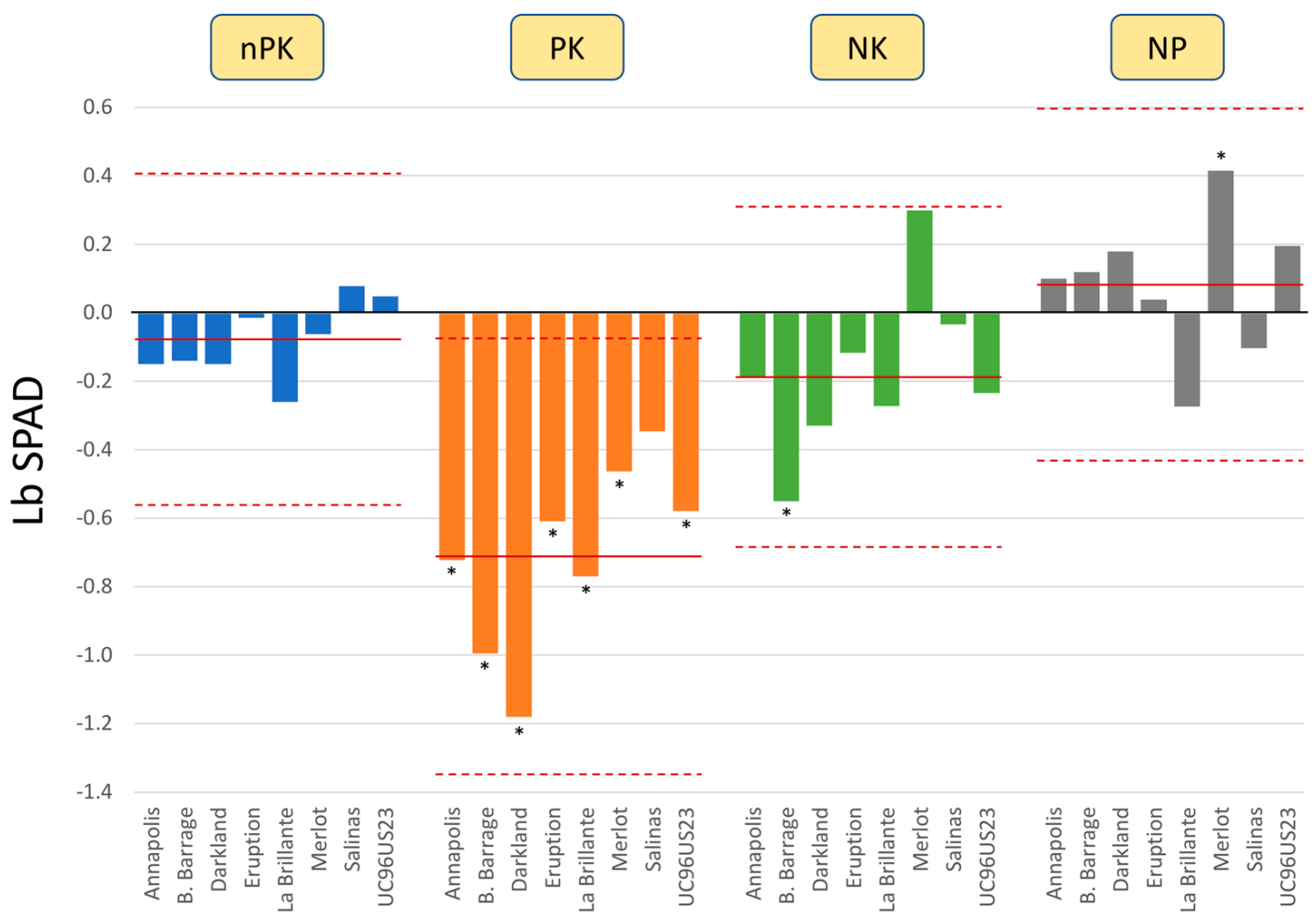

Figure 3. Values of $\Delta_{\mathrm{ABS}}$ and $2 \Delta_{\mathrm{REL}}$ parameters for chlorophyll index (Lb SPAD). The $\Delta_{\mathrm{ABS}}$ bars illustrate (Lb-fold) changes from values observed under control conditions (NPK treatment) for each accession and treatment. NPK treatment (control) supplied optimal concentrations of all three macronutrients, $\mathrm{nPK}$ treatment supplied only $1 / 3$ of $\mathrm{N}$ compared to control, PK treatment did not supply N, NK treatment did not supply P, and NP treatment did not supply K. Significant $(p \leq 0.05) \Delta_{\text {ABS }}$ values detected by Student's $t$-test are indicated by asterisks. Solid horizontal red lines show overall mean of $\Delta_{\mathrm{ABS}}$ at each treatment. Dashed horizontal red lines show $95 \%$ upper and lower decision limits for ANOM. The $2 \Delta_{\text {REL }}$ parameter is calculated as the difference between $\Delta_{\mathrm{ABS}}$ of an accession and the overall mean of $\Delta_{\mathrm{ABS}}$ for all accessions tested for the particular treatment. The significance of the $2 \Delta_{\text {REL }}$ parameter is indicated by capital letters L (significantly lower than overall mean) or $\mathrm{H}$ (significantly higher than overall mean).

\subsection{Nitrogen Content}

Nitrogen content, expressed as a binary logarithm of $\mathrm{N}$ content in $\mathrm{g} \mathrm{kg}^{-1} \mathrm{FW}(\mathrm{Lb} \mathrm{N})$, ranged from 0.28 for 'Darkland' with PK to 2.78 for UC96US23 with NK (Table 3). The overall Lb N mean for accessions was lowest for 'Darkland' (1.50) and highest for UC96US23 (2.18), while the overall mean for treatments ranged from 0.78 with PK to 2.38 with NK (Table 2). Effect sizes for treatments, accessions, and treatment by accession interaction were $\omega^{2}=0.759, \omega^{2}=0.108$, and $\omega^{2}=0.027$, respectively. The $\Delta_{\mathrm{ABS}}$ values were negative and significant for all accessions with $\mathrm{PK}$, for all but two accessions (B. Barrage and UC96US23) at nPK, and 'La Brillante' at NP. In comparison, significant positive $\Delta_{\mathrm{ABS}}$ values, indicating increase in N content, were observed for B. Barrage with NK and NP, 'Darkland' with NK, UC96US23 with NK, and Salinas with NP (Figure 5). Only two $2 \Delta_{\text {REL }}$ values were significant, namely the increase for B. Barrage and decrease for 'Merlot', both with NK. 


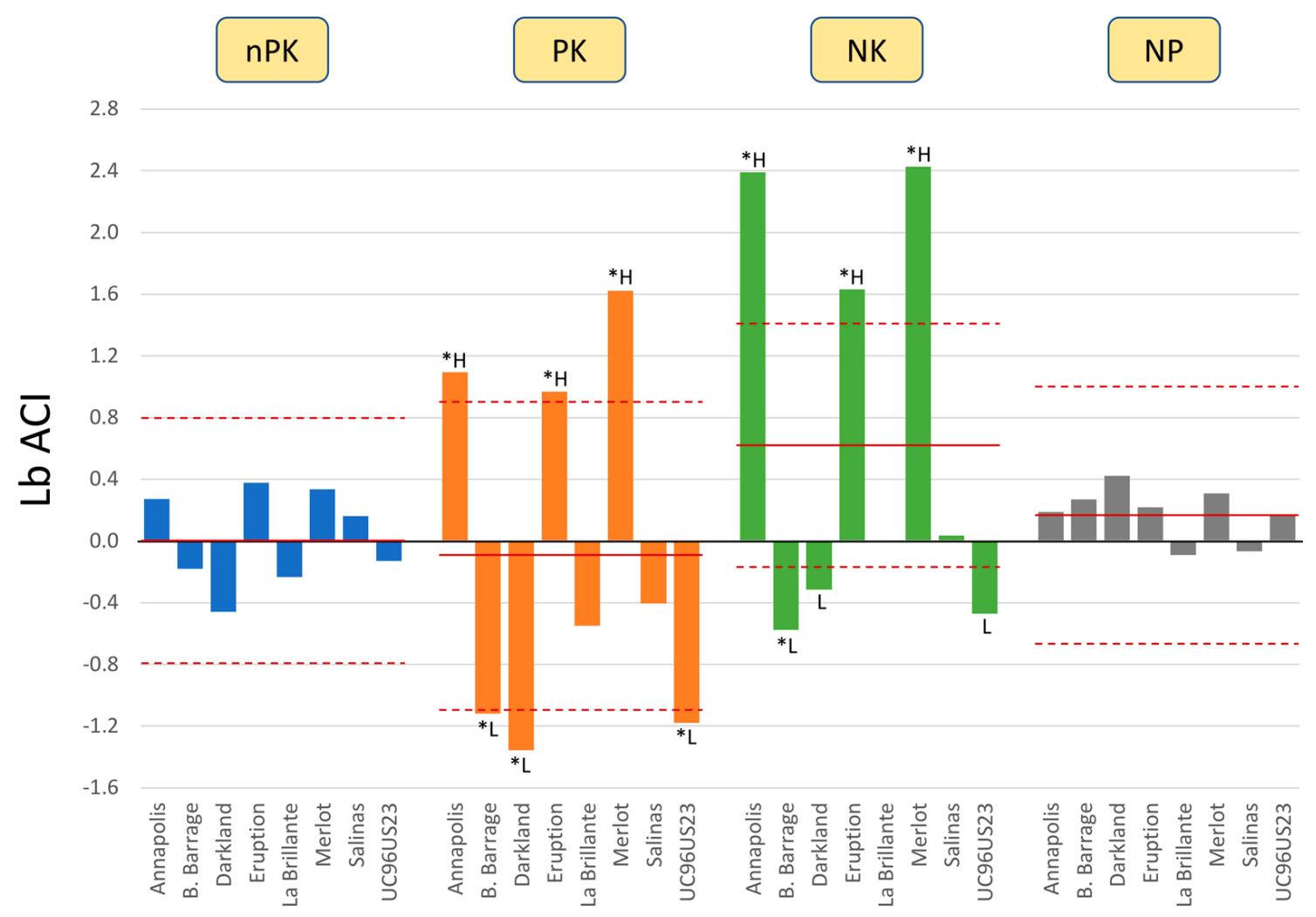

Figure 4. Values of $\Delta_{\mathrm{ABS}}$ and $2 \Delta_{\mathrm{REL}}$ parameters for anthocyanin index (Lb ACI). The $\Delta_{\mathrm{ABS}}$ bars illustrate (Lb-fold) changes from values observed under control conditions (NPK treatment) for each accession and treatment. NPK treatment (control) supplied optimal concentrations of all three macronutrients, nPK treatment supplied only $1 / 3$ of $\mathrm{N}$ compared to control, PK treatment did not supply N, NK treatment did not supply P, and NP treatment did not supply K. Significant $(p \leq 0.05) \Delta_{\text {ABS }}$ values detected by Student's $t$-test are indicated by asterisks. Solid horizontal red lines show overall mean of $\Delta_{\text {ABS }}$ at each treatment. Dashed horizontal red lines show $95 \%$ upper and lower decision limits for ANOM. The $2 \Delta_{\text {REL }}$ parameter is calculated as the difference between $\Delta_{\mathrm{ABS}}$ of an accession and the overall mean of $\Delta_{\mathrm{ABS}}$ for all accessions tested at the particular treatment. The significance of the $2 \Delta_{\mathrm{REL}}$ parameter is indicated by capital letters $\mathrm{L}$ (significantly lower than overall mean) or $\mathrm{H}$ (significantly higher than overall mean).

\subsection{Phosphorus Content}

Phosphorus content, expressed as the binary logarithm of $\mathrm{P}$ content in $\mathrm{g} \mathrm{kg}^{-1} \mathrm{FW}(\mathrm{Lb} \mathrm{P})$, ranged from -3.65 for B. Barrage with NK to -0.21 for 'Merlot' with NPK (Table 3). The overall Lb P mean for accessions was lowest for B. Barrage (-1.65) and highest for 'Merlot' (-1.03), while the overall mean for treatments ranged from -3.18 with NK to -0.53 with NP (Table 2). The effect size was very high for treatments $\left(\omega^{2}=0.892\right)$ and low for accessions $\left(\omega^{2}=0.031\right)$. No effect size was detected for treatment by accession interaction $\left(\omega^{2}=0.000\right)$. Results for effect size matched with those observed for $\Delta_{\mathrm{ABS}}$ and $2 \Delta_{\text {REL }}$ values. All accessions had significant negative $\Delta_{\text {ABS }}$ values with $\mathrm{PK}$ and $\mathrm{NK}$, while none of the $2 \Delta_{\text {REL }}$ values were significant, which indicated that all accessions responded very similarly to treatments (Figure 6). 


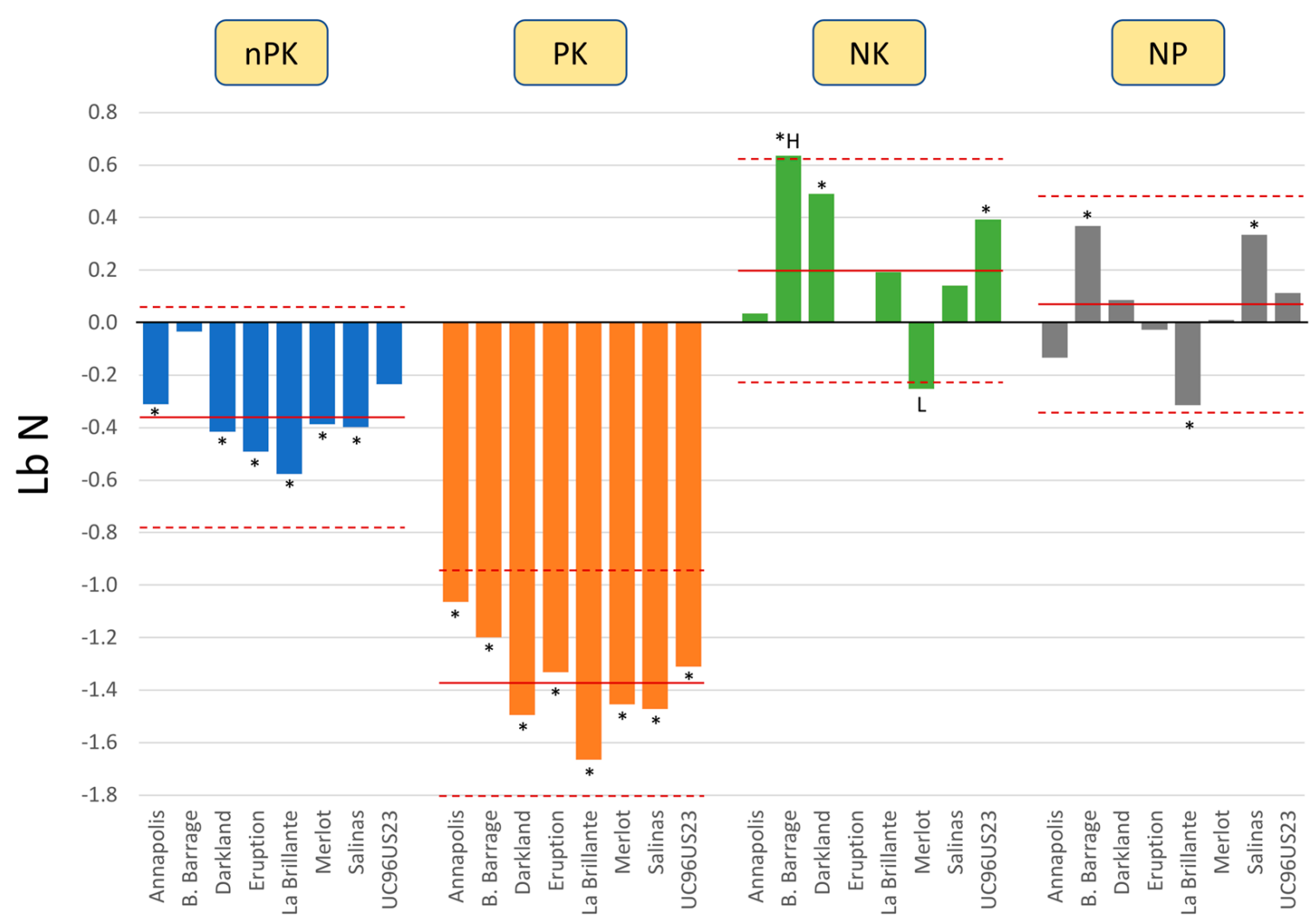

Figure 5. Values of $\Delta_{\mathrm{ABS}}$ and $2 \Delta_{\mathrm{REL}}$ parameters for nitrogen content (Lb N). The $\Delta_{\mathrm{ABS}}$ bars illustrate (Lb-fold) changes from values observed under control conditions (NPK treatment) for each accession and treatment. NPK treatment (control) supplied optimal concentrations of all three macronutrients, nPK treatment supplied only 1/3 of N compared to control, PK treatment did not supply N, NK treatment did not supply P, and NP treatment did not supply K. Significant $(p \leq 0.05) \Delta_{\text {ABS }}$ values detected by Student's $t$-test are indicated by asterisks. Solid horizontal red lines show overall mean of $\Delta_{\text {ABS }}$ at each treatment. Dashed horizontal red lines show $95 \%$ upper and lower decision limits for ANOM. The $2 \Delta_{\text {REL }}$ parameter is calculated as the difference between $\Delta_{\mathrm{ABS}}$ of an accession and the overall mean of $\Delta_{\mathrm{ABS}}$ for all accessions tested for the particular treatment. The significance of the $2 \Delta_{\mathrm{REL}}$ parameter is indicated by capital letters L (significantly lower than overall mean) or $\mathrm{H}$ (significantly higher than overall mean).

\subsection{Potassium Content}

Potassium content, expressed as the binary logarithm of $\mathrm{K}$ content in $\mathrm{g} \mathrm{kg}^{-1} \mathrm{FW}(\mathrm{Lb} \mathrm{K})$, ranged from 0.84 for B. Barrage with NP to 2.60 for 'Eruption' with PK (Table 3). The overall Lb K mean for accessions was lowest for B. Barrage (1.47) and highest for 'Eruption' (2.24), while the overall mean for treatments ranged from 1.36 with NP to 2.28 with nPK (Table 2). The effect size was $\omega^{2}=0.435$ for treatments, $\omega^{2}=0.320$ for accessions, and $\omega^{2}=0.092$ for treatment by accession interaction. Significant negative $\Delta_{\mathrm{ABS}}$ values were observed for all accessions with NP, all but two accessions ('Eruption' and UC96US23) with NK, and four accessions (B. Barrage, 'Darkland', 'La Brillante', and 'Salinas') with PK (Figure 7). Additionally, a significant positive $\Delta_{\mathrm{ABS}}$ was detected, indicating increases in K content compared to control (NPK) treatment were found for 'Darkland' and 'Eruption' with nPK, and for 'Eruption' with PK. Four $2 \Delta_{\text {REL }}$ values were significant at $p<0.05$, two of them were lower than the overall mean (for 'Darkland' and 'La Brillante' with PK), and another two were higher than the overall mean (for 'Eruption' with PK and NK). 


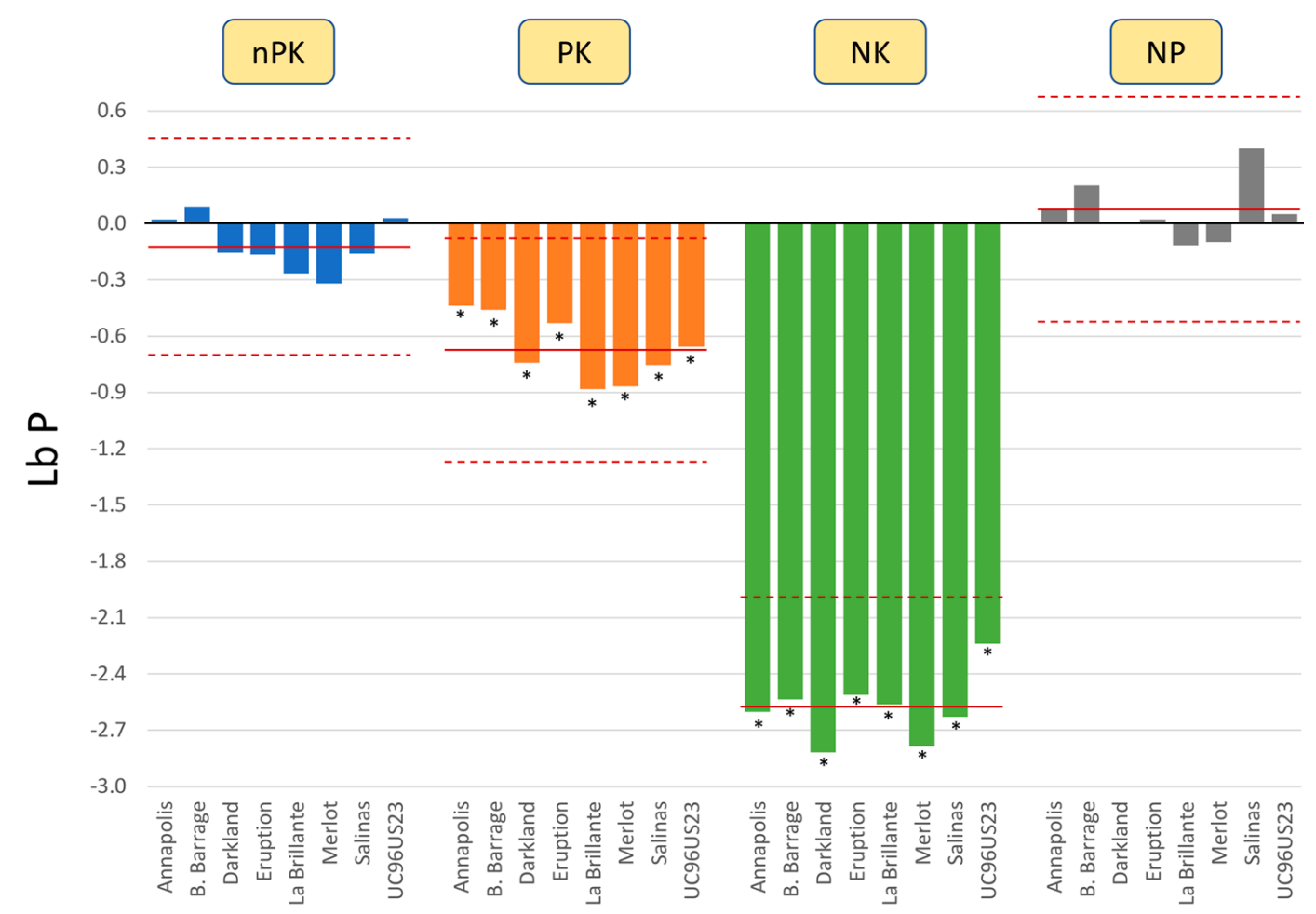

Figure 6. Values of $\Delta_{\mathrm{ABS}}$ and $2 \Delta_{\mathrm{REL}}$ parameters for phosphorus content (Lb P). The $\Delta_{\mathrm{ABS}}$ bars illustrate (Lb-fold) changes from values observed with control (NPK treatment) for each accession and treatment. NPK treatment (control) supplied optimal concentrations of all three macronutrients, nPK treatment supplied only $1 / 3$ of $\mathrm{N}$ compared to control, PK treatment did not supply $\mathrm{N}$, NK treatment did not supply P, and NP treatment did not supply K. Significant $(p \leq 0.05) \Delta_{\text {ABS }}$ values detected by Student's $t$-test are indicated by asterisks. Solid horizontal red lines show the overall mean of $\Delta_{\mathrm{ABS}}$ with each treatment. Dashed horizontal red lines show 95\% upper and lower decision limits for ANOM. The $2 \Delta_{\text {REL }}$ parameter is calculated as the difference between $\Delta_{\mathrm{ABS}}$ of an accession and the overall mean of $\Delta_{\mathrm{ABS}}$ for all accessions tested with the particular treatment. The significance of the $2 \Delta_{\text {REL }}$ parameter is indicated by capital letters L (significantly lower than overall mean) or $\mathrm{H}$ (significantly higher than overall mean).

\subsection{Correlations Between Traits}

Seven of the 15 pairwise, linear correlations between six traits were significant at $p<0.05$ when evaluated on data from all accessions and treatments (Figure 8, Table 4). These positive correlations were found between (listed in descending order of $r$ size) Lb FW and Lb P ( $r=0.56), \mathrm{Lb}$ FW and $\operatorname{Lb} \operatorname{SPAD}(r=0.41), \mathrm{Lb}$ SPAD and Lb N $(r=0.41), \mathrm{Lb}$ FW and Lb N $(r=0.40), \mathrm{Lb}$ SPAD and Lb $\mathrm{ACI}(r=0.30), \mathrm{Lb} \mathrm{N}$ and Lb K $(r=0.19)$, and Lb P and Lb K $(r=0.18)$. When correlations were performed using data from each treatment separately (thus showing correlations across accessions at each treatment), all five correlation coefficients were significant between $\mathrm{Lb} \mathrm{N}$ and $\mathrm{Lb} \mathrm{P}$ ( $r$ from 0.74 to 0.90, Figure 9 ), Lb P and Lb K ( $r$ from 0.72 to 0.88 ), and Lb N and Lb K ( $r$ from 0.62 to 0.97 ). Four of the correlation coefficients were significant between Lb FW and Lb N ( $r$ from -0.43 to -0.54 ), while the fifth correlation with nPK treatment $(r=-0.31)$ was not significant at $P<0.05$. When correlations were performed using data from each accession separately (thus showing correlations across treatments for each accession), all eight correlation coefficients were significant between $\mathrm{Lb}$ FW and $\mathrm{Lb} \mathrm{P}$ ( $r$ from 0.54 to 0.77 ), seven between Lb FW and Lb SPAD (except 'Salinas') and Lb SPAD and Lb N (except 'Salinas'), and six for Lb FW and Lb ACI (three negative for 'Annapolis', 'Eruption', and 'Merlot', and three positive for B. Barrage, 'Darkland', and UC96US23). Frequently, correlations that were significant when calculated from individual treatments were not significant when calculated from individual accessions, and vice versa. 


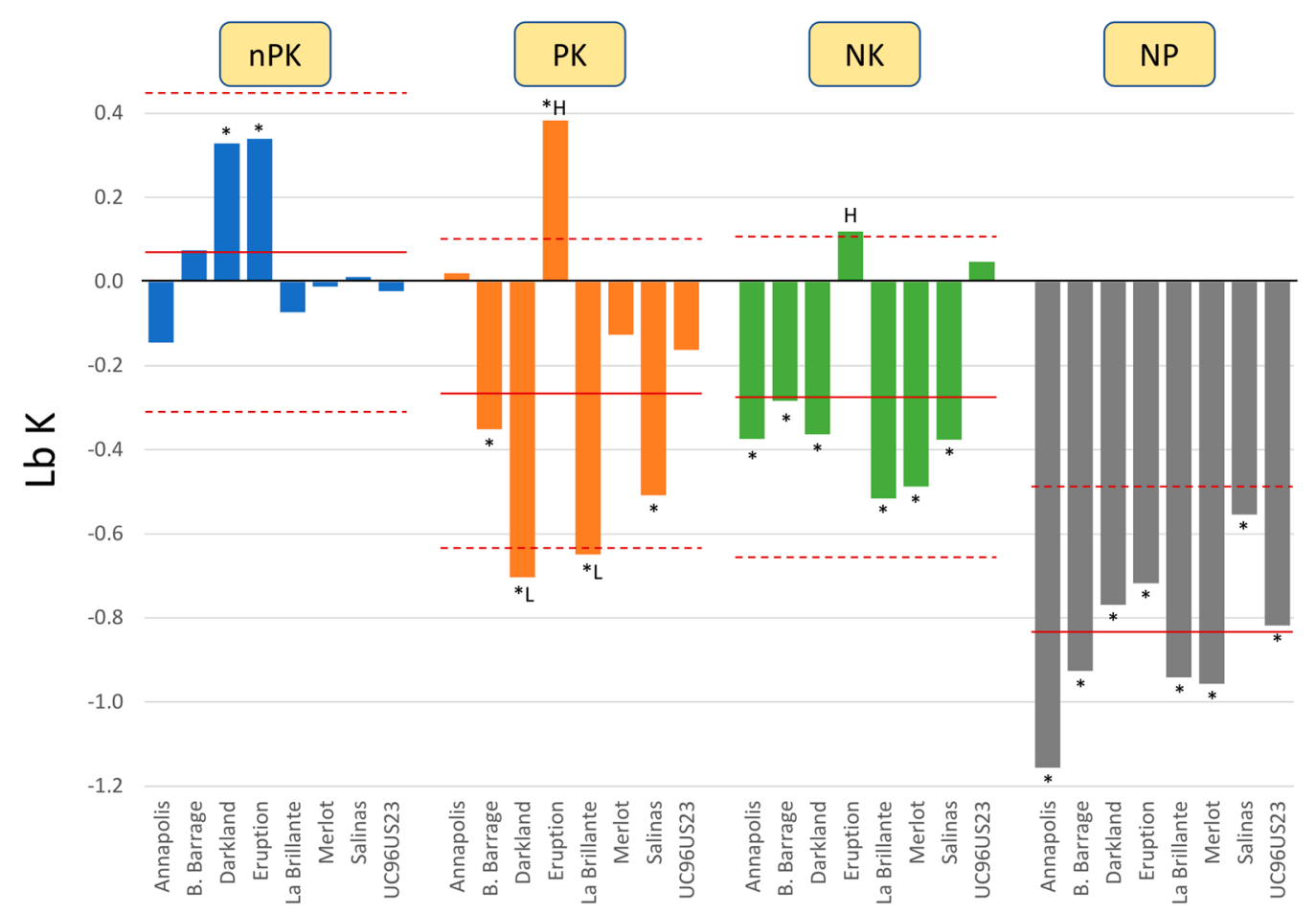

Figure 7. Values of $\Delta_{\mathrm{ABS}}$ and $2 \Delta_{\mathrm{REL}}$ parameters for potassium content (Lb K). The $\Delta_{\mathrm{ABS}}$ bars illustrate (Lb-fold) changes from values observed under control conditions (NPK treatment) for each accession and treatment. NPK treatment (control) supplied optimal concentrations of all three macronutrients, nPK treatment supplied only $1 / 3$ of $\mathrm{N}$ compared to control, PK treatment did not supply N, NK treatment did not supply P, and NP treatment did not supply K. Significant $(p \leq 0.05) \Delta_{\text {ABS }}$ values detected by Student's $t$-test are indicated by asterisks. Solid horizontal red lines show overall mean values of $\Delta_{\text {ABS }}$ with each treatment. Dashed horizontal red lines show $95 \%$ upper and lower decision limits for ANOM. The $2 \Delta_{\text {REL }}$ parameter is calculated as the difference between $\Delta_{\text {ABS }}$ of an accession and the overall mean of $\Delta_{\mathrm{ABS}}$ for all accessions tested with the particular treatment. The significance of the $2 \Delta_{\text {REL }}$ parameter is indicated by capital letters L (significantly lower than overall mean) or $\mathrm{H}$ (significantly higher than overall mean). The missing dashed line for NP is outside of the range indicated on the y-axis. 
Table 4. Pearson correlation coefficients between fresh weight (Lb FW) and content of chlorophyll (Lb SPAD), anthocyanin (Lb ACI), nitrogen (Lb N), phosphorus (Lb $\mathrm{P})$, and potassium ( $\mathrm{Lb} \mathrm{K})$, evaluated from diverse data sets

\begin{tabular}{|c|c|c|c|c|c|c|c|c|c|c|c|c|c|c|c|}
\hline \multirow[b]{2}{*}{ Trait 1} & \multirow[b]{2}{*}{ Trait 2} & \multicolumn{7}{|c|}{ Treatment $^{\mathrm{z}}$} & \multicolumn{5}{|c|}{ Accession $^{\mathrm{y}}$} & \multirow[b]{2}{*}{ Salinas } & \multirow[b]{2}{*}{ UC96US23 } \\
\hline & & All & NPK & nPK & PK & NK & NP & Annapolis & B. Barrage & Darkland & Eruption & La Brillante & Merlot & & \\
\hline $\mathrm{Lb} F W$ & $\begin{array}{c}\text { Lb } \\
\text { SPAD }\end{array}$ & $0.41^{* * x}$ & 0.08 & -0.25 & -0.15 & -0.32 & -0.29 & $0.81^{* *}$ & $0.82 * *$ & $0.78^{* *}$ & $0.58^{* *}$ & $0.57^{* *}$ & $0.45 *$ & 0.44 & $0.79 * *$ \\
\hline Lb FW & Lb ACI & -0.03 & -0.05 & -0.09 & -0.06 & -0.02 & 0.14 & $-0.71^{* *}$ & $0.81 * *$ & $0.75 * *$ & $-0.72 * *$ & 0.44 & $-0.62 * *$ & 0.37 & $0.79 * *$ \\
\hline Lb FW & $\mathrm{Lb} \mathrm{N}$ & $0.40^{* *}$ & $-0.51^{* *}$ & -0.31 & $-0.48^{* *}$ & $-0.54 * *$ & $-0.43^{*}$ & $0.51 *$ & 0.39 & 0.44 & $0.62 * *$ & $0.55^{*}$ & $0.78 * *$ & $0.56 *$ & 0.35 \\
\hline Lb FW & $\mathrm{Lb} P$ & $0.56 * *$ & -0.23 & -0.19 & -0.17 & -0.17 & -0.20 & $0.55 *$ & $0.62 * *$ & $0.58 * *$ & $0.54 *$ & $0.61^{* *}$ & $0.56 *$ & $0.68 * *$ & $0.77^{* *}$ \\
\hline $\mathrm{Lb} F W$ & Lb K & -0.11 & $-0.46^{* *}$ & 0.09 & -0.32 & -0.30 & -0.33 & -0.43 & -0.05 & 0.29 & $-0.52 *$ & 0.21 & -0.14 & 0.43 & -0.23 \\
\hline Lb SPAD & Lb ACI & $0.30 * *$ & $0.54^{* *}$ & 0.34 & 0.10 & $0.69 * *$ & 0.31 & -0.22 & $0.92 * *$ & $0.82 * *$ & -0.14 & $0.80^{* *}$ & 0.30 & $0.69^{* *}$ & $0.85^{* *}$ \\
\hline Lb SPAD & $\mathrm{Lb} \mathrm{N}$ & $0.41 * *$ & -0.28 & -0.18 & 0.17 & -0.20 & 0.05 & $0.65 * *$ & $0.55 *$ & $0.71 * *$ & $0.61^{* *}$ & $0.67^{* *}$ & $0.54 *$ & 0.38 & $0.54 *$ \\
\hline Lb SPAD & $\mathrm{Lb} P$ & 0.09 & $-0.36^{*}$ & -0.21 & -0.13 & -0.29 & 0.04 & 0.11 & 0.42 & 0.24 & 0.14 & 0.21 & -0.16 & -0.03 & 0.38 \\
\hline Lb SPAD & Lb K & -0.14 & $-0.35 *$ & -0.27 & 0.12 & $-0.41 *$ & 0.20 & -0.50 * & -0.08 & 0.25 & -0.26 & 0.42 & -0.55 * & 0.26 & -0.40 \\
\hline Lb ACI & $\mathrm{Lb} N$ & 0.11 & -0.01 & 0.04 & $0.50^{* *}$ & -0.23 & $-0.47^{* *}$ & -0.02 & $0.59 * *$ & $0.75 * *$ & -0.12 & $0.75^{* *}$ & -0.39 & 0.32 & $0.69^{* *}$ \\
\hline $\mathrm{Lb} \mathrm{ACI}$ & $\mathrm{Lb} P$ & -0.11 & 0.01 & 0.11 & $0.49^{* *}$ & -0.11 & 0.09 & $-0.86^{* *}$ & 0.42 & 0.21 & $-0.75^{* *}$ & 0.01 & -0.76 ** & -0.10 & 0.40 \\
\hline Lb ACI & Lb K & 0.04 & -0.18 & -0.20 & $0.62 * *$ & $-0.42 *$ & 0.04 & 0.08 & -0.17 & 0.08 & 0.37 & 0.26 & -0.15 & 0.12 & -0.30 \\
\hline $\mathrm{Lb} N$ & $\mathrm{Lb} P$ & -0.02 & $0.90^{* *}$ & $0.87 * *$ & $0.83^{* *}$ & $0.87^{* *}$ & $0.74^{* *}$ & -0.11 & -0.25 & -0.26 & -0.05 & -0.12 & 0.21 & 0.08 & -0.13 \\
\hline $\mathrm{Lb} N$ & Lb K & $0.19 *$ & $0.97^{* *}$ & $0.62 * *$ & $0.86^{* *}$ & $0.87^{* *}$ & $0.65 * *$ & -0.16 & -0.01 & 0.26 & -0.39 & 0.33 & -0.18 & 0.27 & 0.09 \\
\hline $\mathrm{Lb} P$ & Lb K & $0.18^{*}$ & $0.88^{* *}$ & $0.72 * *$ & $0.77^{* *}$ & $0.81 * *$ & $0.88^{* *}$ & 0.05 & 0.05 & 0.24 & -0.13 & 0.30 & 0.23 & 0.29 & -0.22 \\
\hline
\end{tabular}

${ }^{z}$ NPK treatment (control) supplied optimal concentrations of all three macronutrients, nPK treatment supplied only $1 / 3$ of N compared to control, PK treatment did not supply N, NK
treatment did not supply P, and NP treatment did not supply K. Correlation coefficients were calculated on data from individual treatments across all accessions ( $\mathrm{n}=32$ ), except "all", where correlation coefficients were calculated on complete datasets that included all data $(\mathrm{n}=160)$. ${ }^{\text {y }}$ Correlation coefficients calculated on data from individual accessions across all treatments $(\mathrm{n}=20) .{ }^{\times}$Asterisks indicate correlation coefficients significant at $p \leq 0.05\left(^{*}\right)$ or $p \leq 0.01(* *){ }^{2}$ 


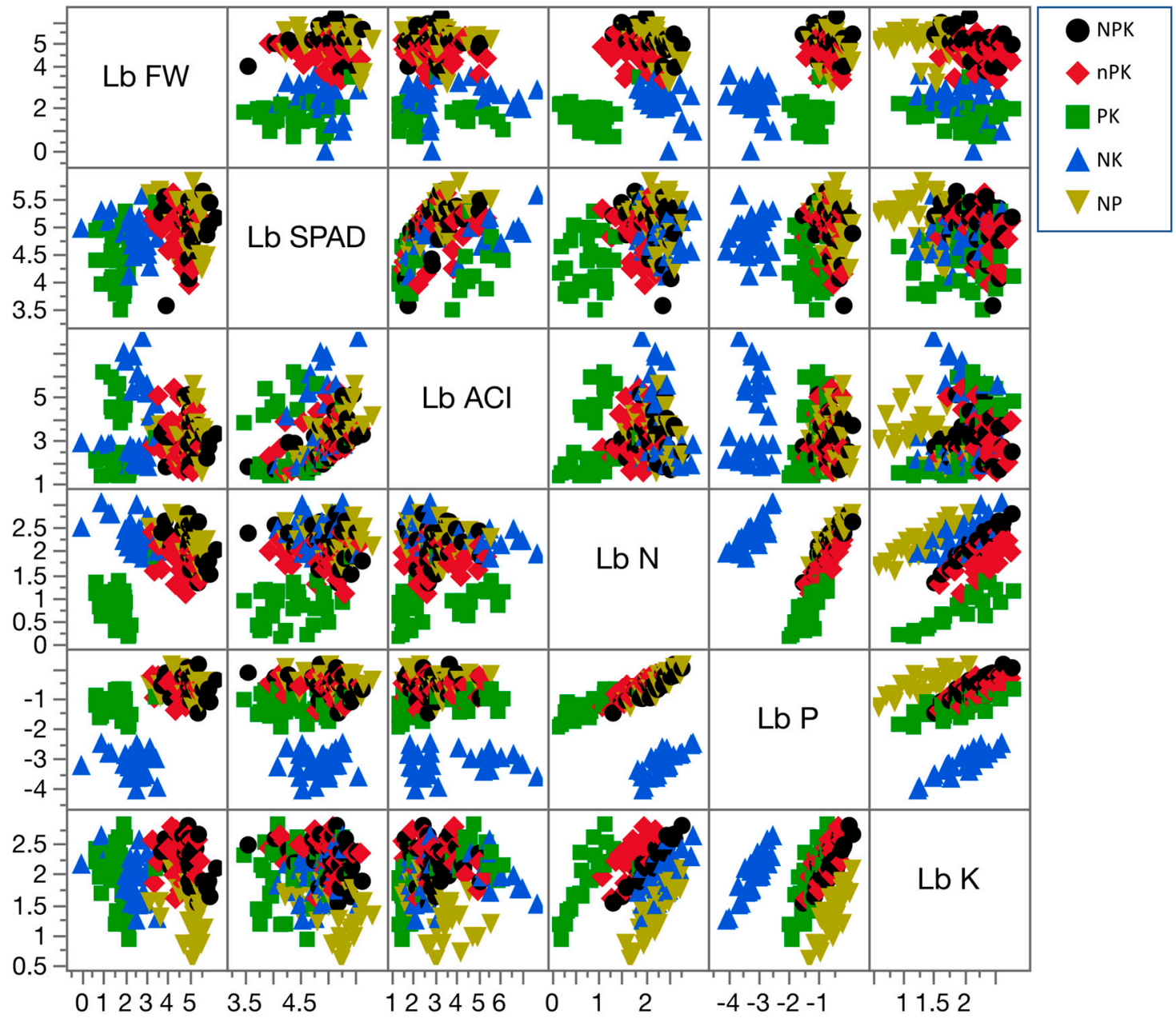

Figure 8. Scatterplot matrix of six traits evaluated in eight accessions in five treatments. Relationships between fresh weight (Lb FW) and content of chlorophyll (Lb SPAD), anthocyanin (Lb ACI), nitrogen $(\mathrm{Lb} \mathrm{N})$, phosphorus ( $\mathrm{Lb} \mathrm{P})$, and potassium (Lb K) are shown in individual windows. Data from five treatments are indicated by icons with different shapes and colors. NPK treatment (control) supplied optimal concentrations of all three macronutrients, nPK treatment supplied only $1 / 3$ of $N$ compared to control, PK treatment did not supply N, NK treatment did not supply P, and NP treatment did not supply K. Note that regressions between traits calculated on data from different fertilizer treatments may have dissimilar slopes or intercepts but similar correlation coefficients (Table 4).

\section{Discussion}

Commercial lettuce production requires adequate levels of NPK to provide high yield and good product quality. The composition of lettuce accessions tested in this study depended on macronutrients used in each treatment (Tables 2 and 3). Generally, untransformed values (provided in Supplementary Table S1) for SPAD, ACI, N content, P content, and K content matched well with those previously reported for lettuce [3,4,30-32]. Fresh weight and SPAD values significantly decreased at low rates of $\mathrm{N}(\mathrm{PK})$ and $\mathrm{P}(\mathrm{NK})$, but not K (NP) (Table 2). These results agree with those observed for baby spinach, where both biomass yield and chlorophyll content increased significantly with increasing $\mathrm{N}$ and $\mathrm{P}$ rates, although not with increasing rates of K [14]. Similarly, chlorophyll levels in lettuce increased linearly with $\mathrm{N}$ rate from 0 to $960 \mathrm{mg}$ per $\mathrm{kg}$ of soil [15].

The overall increase in anthocyanin content (Lb ACI) was associated with phosphorus deprivation with NK treatment (Figure 4). This observation corroborates previous results obtained with diverse plant species, such as petunia (Petunia $\times$ hybrida hort. ex E. Vilm.) [33], maize (Zea mays L.) [17], and cabbage (Brassica oleracea L. var. capitata) [18], where higher anthocyanin production was related to 
phosphorus deficiency. Besides phosphorus deficiency, nitrogen deficiency can also lead to higher anthocyanin accumulation [16-18]. Results from this study validate the effect of nitrogen deficiency on increased accumulation of anthocyanin. However, current results also point out differences among tested accessions in their reaction to $\mathrm{P}$ and $\mathrm{N}$ deprivation (Figure 4). While accessions that appeared as dark-red ('Annapolis' and 'Merlot') and red ('Eruption') colored in control treatment conditions (NPK) all responded to both $\mathrm{P}$ and $\mathrm{N}$ deprivation by increasing their anthocyanin content, accessions that appeared dark green in control conditions (B. Barrage, 'Darkland', and UC96US23) had lower accumulation of anthocyanin than other tested accessions when deprived of $\mathrm{P}$ or $\mathrm{N}$. Accessions that were light green ('La Brillante') or green ('Salinas') in control conditions only had minimal changes in their content of anthocyanin in NK and PK treatments. Differences among accessions in their accumulation of anthocyanin under stress conditions were previously observed under both high and low temperature conditions [34]. At low temperature $\left(3^{\circ} \mathrm{C}\right)$, accumulation of anthocyanin increased as compared to optimal temperature $\left(21^{\circ} \mathrm{C}\right)$, but only in red-colored accessions and not in green ones. In comparison, under high temperature $\left(39^{\circ} \mathrm{C}\right)$, anthocyanin accumulation decreased in red-colored accessions and increased in all that were green-colored [34]. Because several genes are involved in controlling red leaf color in lettuce [35-37], it is possible that these genes respond differently to different environmental stimuli. To better understand the regulatory mechanism of anthocyanin biosynthesis in lettuce and investigate why plants with high levels of anthocyanin content responded differently to nutrient stresses, further studies using $\mathrm{N}$ and $\mathrm{P}$ deficiency treatments are needed.

Correlation coefficients calculated with the complete dataset (all accessions and all fertilizer treatments) frequently differed from those determined from either individual accessions tested across all treatments or from individual treatments that included all accessions (Figure 9). Such differences in results emphasize the importance of using appropriate experimental design when evaluating correlations between traits. For example, a significant positive correlation detected between $\mathrm{Lb} \mathrm{N}$ and Lb SPAD corroborated the previously reported strong relationship between concentration of $\mathrm{N}$ and chlorophyll (as determined by SPAD) [38]. It is possible that such a strong relationship exists because up to $80 \%$ of leaf nitrogen is allocated in chloroplasts [39]. However, significant correlation between the two traits was detected only when performed on data from individual accessions tested in multiple environments (treatments), but not when data from all accessions tested in a single treatment were used for analysis. Comparable results were previously observed for rice (Oryza sativa L.) plants tested at different developmental stages [40]. Coefficients of determination between SPAD values and leaf nitrogen concentration at each of the four developmental stages ranged from $R^{2}=0.61$ to 0.88 , but decreased to only $R^{2}=0.11$ when data from all developmental stages were pooled [40].

Strong, positive, linear correlations were detected between values of $\mathrm{N}, \mathrm{P}$, and $\mathrm{K}(\mathrm{Lb} \mathrm{N}, \mathrm{Lb} \mathrm{P}$, and $\mathrm{Lb} \mathrm{K}$ ) when tested in individual treatments, but not when tested using individual accessions. Previously, significant positive correlations were found between N, P, and K contents in grains of rice ( $r$ from 0.89 to 0.97 ) [41] and in plantlets of stevia (Stevia rebaudiana Bertoni) ( $r$ from 0.59 to 0.85) [42], indicating that uptake and accumulation of these compounds may be related. It needs to be emphasized, however, that the strong correlations detected between contents of $\mathrm{N}, \mathrm{P}$, and $\mathrm{K}$ in the present study were determined on a fresh weight basis. It is possible that calculations performed on a dry weight basis would provide somewhat different results. 


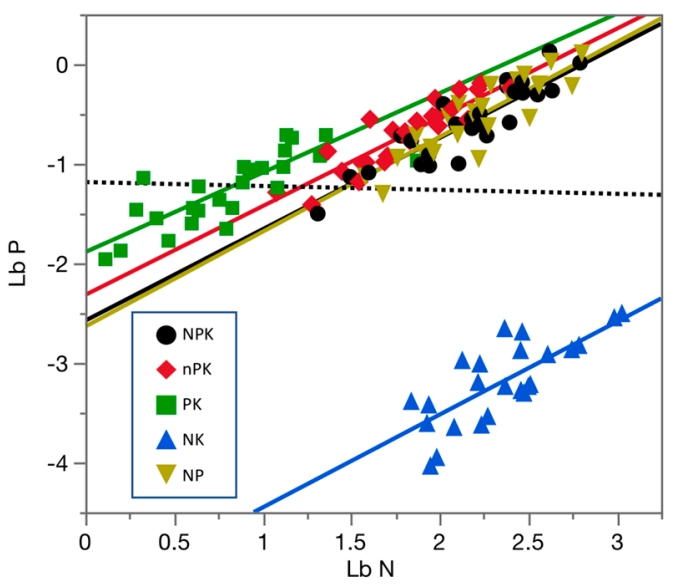

Figure 9. Correlation between the content of nitrogen $(\mathrm{Lb} \mathrm{N})$ and phosphorus $(\mathrm{Lb} \mathrm{P})$ in baby leaf lettuce tested with different fertilizer treatments. Strong, positive, linear correlations ranging from $r=0.74$ to $r=0.90(p<0.01$, Table 4$)$ were detected between $\mathrm{Lb} \mathrm{N}$ and $\mathrm{Lb} \mathrm{P}$ with each of the five treatments when analyzed separately. The correlation coefficient dropped to $r=-0.02$ (black, dashed line) when data from all treatments were considered together. Five treatments are indicated by icons with different shapes and colors. NPK treatment (control) supplied optimal concentrations of all three macronutrients, nPK treatment supplied only $1 / 3$ of $\mathrm{N}$ compared to control, PK treatment did not supply N, NK treatment did not supply $\mathrm{P}$, and NP treatment did not supply K.

Use of $\Delta_{\mathrm{ABS}}$ and $2 \Delta_{\mathrm{REL}}$ parameters allowed the identification of accessions with significantly changed values (performance) with different treatments. While the $\Delta_{\mathrm{ABS}}$ parameter indicated an absolute change in performance of a particular accession, the $2 \Delta_{\text {REL }}$ parameter indicated a relative change compared to other tested accessions. The difference between the two approaches can be seen, for example, by the decrease of fresh weight (Lb FW) with PK treatment (Figure 2). All accessions had a significant decrease in their $\Delta_{\mathrm{ABS}}$ values; however, only 'Darkland' had a significant decrease in its $2 \Delta_{\text {REL }}$ value, indicating that the fresh weight of this accession decreased more than the overall average of other accessions. Another example is the change in potassium content $(\mathrm{Lb} \mathrm{K})$ with NK treatment for 'Eruption' (Figure 7). Although a change was not detected when $\Delta_{\mathrm{ABS}}$ was tested, the $2 \Delta_{\mathrm{REL}}$ value was higher than the overall mean. Using the $2 \Delta_{\text {REL }}$ parameter as an indicator, a decrease was detected for 'Darkland' fresh weight with PK, 'Merlot' nitrogen content with NK, 'Darkland' and 'La Brillante' potassium content with PK, and B. Barrage, 'Darkland', and UC96US23 anthocyanin content with both PK and NK. On the other hand, higher than overall values of $2 \Delta_{\text {REL }}$ were found for B. Barrage nitrogen content with NK, 'Eruption' potassium content with both PK and NK, and 'Annapolis', 'Eruption', and 'Merlot' anthocyanin content with both PK and NK (Figures 2-7). Values of $\Delta_{\mathrm{ABS}}$ and $2 \Delta_{\mathrm{REL}}$ can, thus, be used to identify over- or underperforming accessions. These two approaches complement each other by helping to find lettuce accessions with absolute or relative (Lb-fold) changes in their performance. Results can then be used to select accessions with the most desirable traits. Additional accessions of both cultivated and prickly lettuce should be tested using different fertilizer treatments to identify genotypes for which the composition is minimally affected by decreased fertilization with $\mathrm{N}, \mathrm{P}$, and K. Such genotypes could then be used to study nitrogen, phosphorus, and potassium accumulation in lettuce and to develop novel cultivars with improved efficiency of absorption and use of these macronutrients. Improving the efficiency of N, P, and K utilization would lead to more economic production of lettuce, while enhancing environmental sustainability.

\section{Conclusions}

Results of the present study were as follows. 
Potassium deprivation had no effect (or no consistent effect across lettuce accessions) on any of the tested traits, with the exception of potassium content in fresh tissue, which substantially and significantly decreased.

Nitrogen and phosphorus deprivations extensively decreased fresh weight and affected plant composition.

The largest effects of treatments were observed on content of phosphorus and fresh weight; the largest effects of accessions were detected on anthocyanin content; and the largest effects of interactions were also found for anthocyanin content.

In phosphorus and nitrogen deprivation treatments, anthocyanin substantially increased in red-colored accessions, but decreased in those that were dark green-colored, indicating differences in the regulatory mechanism(s) of anthocyanin biosynthesis.

Potassium content in 'Eruption' lettuce increased with nitrogen and phosphorus deprivation treatments.

Correlations between traits were substantially affected by selection of datasets used for analyses. Some of the correlations were very high (e.g., between content of nitrogen, phosphorus, and potassium) when individual treatments were analyzed separately but not with combined data, while others were more likely to be detected when analyzed with individual accessions (e.g., between fresh weight and anthocyanin content, although in opposite directions with different accessions).

Parameters $\Delta_{\mathrm{ABS}}$ and $2 \Delta_{\mathrm{REL}}$, as described in this study, allow the detection of over- or underperforming accessions. These two parameters indicated Lb-fold changes in absolute and relative performance of an accession, were complementary to each other, and should be considered jointly.

Supplementary Materials: The following are available online at http://www.mdpi.com/2311-7524/6/1/15/s1. Table S1: Original measured values of fresh weight, and content of chlorophyll, anthocyanin, nitrogen, phosphorus, and potassium for eight lettuce accessions tested with five fertilizer treatments and harvested at baby leaf stage.

Funding: This research received no external funding.

Acknowledgments: The author would like to thank L. Rosental for excellent technical assistance in various phases of this research.

Conflicts of Interest: The author declares no conflict of interest.

Disclaimer: The mentioning of trade names or commercial products in this publication is solely for the purpose of providing specific information and does not imply recommendation or endorsement by the U.S. Department of Agriculture (USDA).

\section{References}

1. Simko, I.; Hayes, R.J.; Mou, B.; McCreight, J.D. Lettuce and Spinach. In Yield Gains in Major U.S. Field Crops; CSSA Special Publications; Smith, S., Diers, B., Specht, J., Carver, B., Eds.; American Society of Agronomy, Inc., Crop Science Society of America, Inc., Soil Science Society of America, Inc.: Madison, WI, USA, 2014; pp. 53-86.

2. Saini, R.K.; Ko, E.Y.; Keum, Y.-S. Minimally processed ready-to-eat baby-leaf vegetables: Production, processing, storage, microbial safety, and nutritional potential. Food Rev. Int. 2017, 33, 644-663. [CrossRef]

3. Mou, B. Nutrient content of lettuce and its improvement. Curr. Nutr. Food Sci. 2009, 5, 242-248. [CrossRef]

4. Simko, I. Genetic variation and relationship among content of vitamins, pigments, and sugars in baby leaf lettuce. Food Sci. Nutr. 2019, 7, 3317-3326. [CrossRef] [PubMed]

5. Sytar, O.; Zivcak, M.; Bruckova, K.; Brestic, M.; Hemmerich, I.; Rauh, C.; Simko, I. Shift in accumulation of flavonoids and phenolic acids in lettuce attributable to changes in ultraviolet radiation and temperature. Sci. Hortic. 2018, 239, 193-204. [CrossRef]

6. Dillard, C.J.; German, J.B. Phytochemicals: Nutraceuticals and human health. J. Sci. Food Agric. 2000, 80, 1744-1756. [CrossRef] 
7. Becker, C.; Urlić, B.; Špika, M.J.; Kläring, H.-P.; Krumbein, A.; Baldermann, S.; Ban, S.G.; Perica, S.; Schwarz, D. Nitrogen limited red and green leaf lettuce accumulate flavonoid glycosides, caffeic acid derivatives, and sucrose while losing chlorophylls, $\beta$-carotene and xanthophylls. PLOS ONE 2015, 10, e0142867. [CrossRef]

8. Gruda, N.; Savvas, D.; Colla, G.; Rouphael, Y. Impacts of genetic material and current technologies on product quality of selected greenhouse vegetables-A review. Eur. J. Hortic. Sci. 2018, 83. [CrossRef]

9. Hoque, M.M.; Ajwa, H.; Othman, M.; Smith, R.; Cahn, M. Yield and postharvest quality of lettuce in response to nitrogen, phosphorus, and potassium fertilizers. HortScience 2010, 45, 1539-1544. [CrossRef]

10. Mampholo, B.M.; Maboko, M.; Soundy, P.; Sivakumar, D. Postharvest responses of hydroponically grown lettuce varieties to nitrogen application rate. J. Integr. Agric. 2019, 18, 2272-2283. [CrossRef]

11. Lecompte, F.; Abro, M.; Nicot, P. Can plant sugars mediate the effect of nitrogen fertilization on lettuce susceptibility to two necrotrophic pathogens: Botrytis cinerea and Sclerotinia sclerotiorum? Plant Soil 2013, 369, 387-401. [CrossRef]

12. Vorholt, J.A. Microbial life in the phyllosphere. Nat. Rev. Microbiol. 2012, 10, 828. [CrossRef] [PubMed]

13. Brandl, M.; Amundson, R. Leaf age as a risk factor in contamination of lettuce with Escherichia coli O157: H7 and Salmonella enterica. Appl. Environ. Microbiol. 2008, 74, 2298-2306. [CrossRef] [PubMed]

14. Nemadodzi, L.E.; Araya, H.; Nkomo, M.; Ngezimana, W.; Mudau, N.F. Nitrogen, phosphorus, and potassium effects on the physiology and biomass yield of baby spinach (Spinacia oleracea L.). J. Plant Nutr. 2017, 40, 2033-2044. [CrossRef]

15. Fontes, P.; Pereira, P.; Conde, R. Critical chlorophyll, total nitrogen, and nitrate-nitrogen in leaves associated to maximum lettuce yield. J. Plant Nutr. 1997, 20, 1061-1068. [CrossRef]

16. Boldt, J.K.; Meyer, M.H.; Erwin, J.E. Foliar anthocyanins: A horticultural review. Hortic. Rev. (Am. Soc. Hortic. Sci.) 2014, 42, 209-252.

17. Lawanson, A.; Akindele, B.; Fasalojo, P.; Akpe, B. Time-course of anthocyanin formation during deficiencies of nitrogen, phosphorus and potassium in seedlings of Zea mays Linn. var. ES 1. Z. Pflanzenphysiol. 1972, 66, 251-253. [CrossRef]

18. Yuan, Y.; Chiu, L.-W.; Li, L. Transcriptional regulation of anthocyanin biosynthesis in red cabbage. Planta 2009, 230, 1141-1153. [CrossRef]

19. White, P.J.; Brown, P.H. Plant nutrition for sustainable development and global health. Ann. Bot. 2010, 105, 1073-1080. [CrossRef]

20. Verzeaux, J.; Hirel, B.; Dubois, F.; Lea, P.J.; Tétu, T. Agricultural practices to improve nitrogen use efficiency through the use of arbuscular mycorrhizae: Basic and agronomic aspects. Plant Sci. 2017, 264, 48-56. [CrossRef]

21. Parry, C.; Blonquist, J.M., Jr.; Bugbee, B. In situ measurement of leaf chlorophyll concentration: Analysis of the optical/absolute relationship. Plant Cell Environ. 2014, 37, 2508-2520. [CrossRef]

22. Van den Berg, A.K.; Perkins, T.D. Nondestructive estimation of anthocyanin content in autumn sugar maple leaves. HortScience 2005, 40, 685-686. [CrossRef]

23. Miller, R.O. Extractable Chloride, Nitrate, Orthophosphate, Potassium, and Sulfate-Sulfur in Plant Tissue: 2\% Acetic Acid Extraction. In Handbook of Reference Methods for Plant Analysis; Kalra, Y.P., Ed.; CRC Press: Boca Raton, FL, USA, 1998; pp. 115-118.

24. Prokopy, W.R. Phosphorus in Acetic Acid Extracts; Lachat Instruments: Milwaukee, WI, USA, 1995.

25. Jones, B.J.J. Extraction of Chloride (Cl), Nitrate (NO3), Orthophosphate (PO4), Potassium (K), and Sulfate (SO4) from Plant Tissue Using 2\% Acetic Acid. In Laboratory Guide for Conducting Soil Tests and Plant Analysis; CRC Press: Boca Raton, FL, USA, 2001; pp. 228-229.

26. Nakagawa, S.; Cuthill, I.C. Effect size, confidence interval and statistical significance: A practical guide for biologists. Biol. Rev. 2007, 82, 591-605. [CrossRef]

27. Poorter, H.; Garnier, E. Plant growth analysis: An evaluation of experimental design and computational methods. J. Exp. Bot. 1996, 47, 1343-1351. [CrossRef]

28. Hoffmann, W.A.; Poorter, H. Avoiding bias in calculations of relative growth rate. Ann. Bot. 2002, 90, 37-42. [CrossRef] [PubMed]

29. Kerkhoff, A.J.; Enquist, B. Multiplicative by nature: Why logarithmic transformation is necessary in allometry. J. Theor. Biol. 2009, 257, 519-521. [CrossRef] 
30. Johnstone, P.; Hartz, T.; Cahn, M.; Johnstone, M. Lettuce response to phosphorus fertilization in high phosphorus soils. HortScience 2005, 40, 1499-1503. [CrossRef]

31. Mampholo, B.M.; Maboko, M.M.; Soundy, P.; Sivakumar, D. Phytochemicals and overall quality of leafy lettuce (Lactuca sativa L.) varieties grown in closed hydroponic system. J. Food Qual. 2016, 39, 805-815. [CrossRef]

32. Zhang, G.; Johkan, M.; Hohjo, M.; Tsukagoshi, S.; Maruo, T. Plant growth and photosynthesis response to low potassium conditions in three lettuce (Lactuca sativa) types. Hortic. J. 2017. [CrossRef]

33. Hosokawa, M.; Yamauchi, T.; Takahama, M.; Goto, M.; Mikano, S.; Yamaguchi, Y.; Tanaka, Y.; Ohno, S.; Koeda, S.; Doi, M. Phosphorus starvation induces post-transcriptional CHS gene silencing in Petunia corolla. Plant Cell Rep. 2013, 32, 601-609. [CrossRef]

34. Simko, I.; Hayes, R.J.; Furbank, R.T. Non-destructive phenotyping of lettuce plants in early stages of development with optical sensors. Front. Plant Sci. 2016, 7, 1985. [CrossRef]

35. Gurdon, C.; Poulev, A.; Armas, I.; Satorov, S.; Tsai, M.; Raskin, I. Genetic and phytochemical characterization of lettuce flavonoid biosynthesis mutants. Sci. Rep. 2019, 9, 3305. [CrossRef] [PubMed]

36. Mamo, B.E.; Hayes, R.J.; Truco, M.J.; Puri, K.D.; Michelmore, R.W.; Subbarao, K.V.; Simko, I. The genetics of resistance to lettuce drop (Sclerotinia spp.) in lettuce in a recombinant inbred line population from Reine des Glaces $\times$ Eruption. Theor. Appl. Genet. 2019, 132, 2439-2460. [CrossRef] [PubMed]

37. Su, W.; Tao, R.; Liu, W.; Yu, C.; Yue, Z.; He, S.; Lavelle, D.; Zhang, W.; Zhang, L.; An, G. Characterization of four polymorphic genes controlling red leaf colour in lettuce that have undergone disruptive selection since domestication. Plant Biotechnol. J. 2020, 18, 479-490. [CrossRef] [PubMed]

38. Ercoli, L.; Mariotti, M.; Masoni, A.; Massantini, F. Relationship between nitrogen and chlorophyll content and spectral properties in maize leaves. Eur. J. Agron. 1993, 2, 113-117. [CrossRef]

39. Makino, A.; Osmond, B. Effects of nitrogen nutrition on nitrogen partitioning between chloroplasts and mitochondria in pea and wheat. Plant Physiol. 1991, 96, 355-362. [CrossRef]

40. Wang, Y.; Wang, D.; Shi, P.; Omasa, K. Estimating rice chlorophyll content and leaf nitrogen concentration with a digital still color camera under natural light. Plant Methods 2014, 10, 36. [CrossRef]

41. Lavakush; Yadav, J.; Verma, J.P.; Jaiswal, D.K.; Kumar, A. Evaluation of PGPR and different concentration of phosphorus level on plant growth, yield and nutrient content of rice (Oryza sativa). Ecol. Eng. 2014, 62, 123-128. [CrossRef]

42. Vafadar, F.; Amooaghaie, R.; Otroshy, M. Effects of plant-growth-promoting rhizobacteria and arbuscular mycorrhizal fungus on plant growth, stevioside, NPK, and chlorophyll content of Stevia rebaudiana. J. Plant Interact. 2014, 9, 128-136. [CrossRef] 